Article

\title{
Increase in PKC $\alpha$ Activity during Heart Failure Despite the Stimulation of PKC $\alpha$ Braking Mechanism
}

\author{
Naveed Aslam 1,2 \\ 1 BioSystOmics 4424 Jim West Street, Bellaire, TX 77401, USA; naveed.aslam@biosystomics.com \\ 2 Department of Chemistry \& Chemical Engineering, Lahore University of Management Sciences (LUMS), \\ Lahore 54792, Pakistan
}

Received: 29 February 2020; Accepted: 3 April 2020; Published: 7 April 2020

\begin{abstract}
Rationale: Heart failure (HF) is marked by dampened cardiac contractility. A mild therapeutic target that improves contractile function without desensitizing the $\beta$-adrenergic system during HF may improve cardiac contractility and potentially survival. Inhibiting protein kinase $\mathrm{C} \alpha$ (PKC $\alpha$ ) activity may fit the criteria of a therapeutic target with milder systemic effects that still boosts contractility in HF patients. PKC $\alpha$ activity has been observed to increase during HF. This increase in PKC $\alpha$ activity is perplexing because it is also accompanied by up-regulation of a molecular braking mechanism. Objective: I aim to explore how PKC $\alpha$ activity can be increased and maintained during HF despite the presence of a molecular braking mechanism. Methods and Results: Using a computational approach, I show that the local diacylglycerol (DAG) signaling is regulated through a two-compartment signaling system in cardiomyocytes. These results imply that after massive myocardial infarction (MI), local homeostasis of DAG signaling is disrupted. The loss of this balance leads to prolonged activation of PKC $\alpha$, a key molecular target linked to LV remodeling and dysfunctional filling and ejection in the mammalian heart. This study also proposes an explanation for how DAG homeostasis is regulated during normal systolic and diastolic cardiac function. Conclusions: I developed a novel two-compartment computational model for regulating DAG homeostasis during Ang II-induced heart failure. This model provides a promising tool with which to study mechanisms of DAG signaling regulation during heart failure. The model can also aid in identification of novel therapeutic targets with the aim of improving the quality of life for heart failure patients.
\end{abstract}

Keywords: myocardial infarction; translocation; lipid; contractile dysfunction; $\beta$-adrenergic system desensitization

\section{Introduction}

Shortness of breath and angina are may be the first significant clinical symptoms of coronary artery disease (CAD) [1]. CAD may lead to myocardial infarction (MI). MI can induce structural remodeling [2] in both the left and right ventricles, though it affects the left ventricle more frequently. Clinically, cardiac remodeling manifests through changes in the size, shape, and function of the heart [2,3]. Remodeling events are characterized by complex architectural alterations that result in rearrangement of the existing myocardial structure. These changes take place at both infarcted and non-infarcted areas of the myocardium [2,3]. These alterations include dilation of the ventricular cavity, myocyte necrosis, thinning of the infarcted myocardium, and interstitial fibrosis [1-5]. After MI, early ventricular remodeling is a compensatory adaptive response to loss of function in the myocardium. The architectural alterations following MI can, in turn, preserve cardiac performance, at least in the early phase immediately following infarction. However, these initial compensatory changes can transition into permanent modifications of the cardiac structure leading to heart failure [1-5]. Despite significant progress in our understanding of the cellular and molecular basis of cardiac remodeling, 
the molecular mechanisms responsible for the transformation of initial compensatory responses into responses that impair cardiac function remain elusive [5].

The transition from compensatory response to loss of function can partially be linked to impaired calcium $\left(\mathrm{Ca}^{+2}\right)$ homeostasis [5,6]. Clinically, dysregulation of calcium signaling manifests as contractile dysfunction and the development of malignant ventricular arrhythmia [5,6]. Experimental observations in angiotensin II (Ang II)-stimulated cardiomyocytes show that, in part, calcium homeostasis is regulated through the G $\alpha$ q-DAG-PKC $\alpha-D G K \zeta$ signaling cascade [5-7]. PKC $\alpha$ is a key molecule linked to $\mathrm{G} \alpha \mathrm{q}$-induced heart failure [8]. PKC $\alpha$ belongs to the conventional protein kinase $\mathrm{C}$ (cPKC) family of serine/threonine protein kinases. These kinases are canonically activated by $\mathrm{Ca}^{+2}$ and lipid signaling [9]. PKC $\alpha$ is the most prominent member of the PKC family and is expressed in mouse, human and rabbit heart tissue [8,9]. Previous observations have linked PKC $\alpha$ to impaired left ventricular filling and ejection during heart failure. PKC $\alpha$ is necessary and sufficient to induce ventricular systolic and diastolic dysfunction [8,9]. Pharmacological and genetic inhibition of $\mathrm{PKC} \alpha$ clearly improve contractility during heart failure, attenuating the extent of damage and disease [8,9]. Prolonged activation of PKC $\alpha$ may lead to serious malignant outcomes by inducing systolic and diastolic dysfunction [8-12].

DGK $\zeta$ is another key functional effector that signals during G $\alpha q$-induced heart failure [13]. Previous research has shown that cardiac-specific overexpression of DGK $\zeta$ suppresses remodeling and fibrosis in the left ventricle (LV) independent of hemodynamic regulation. Thus, overexpression of DGK $\zeta$ under these conditions rescues angiotensin-induced congestive heart failure [13]. Previous data indicates that overexpression of DGK $\zeta$ also improves survival after MI [14]. Both DGK $\zeta$ and PKC $\alpha$ have been individually linked to angiotensin II/G $\alpha$-induced cardiac dysfunction, though expression of the two molecules results in opposite outcomes [13-16]. PKC $\alpha$ appears to be a negative regulator, whereas DGK $\zeta$ appears to positively regulate the preservation of left ventricular function after MI [9-16]. Some observations indicate that DGK $\zeta$ can prevent angiotensin II-induced activation of PKC $\alpha$ and subsequent contractile dysfunction [13-18]. The precise molecular details of the cardiac-specific protective function of DGK $\zeta$, however, remains elusive. Recent experimental evidence from multiple cell types indicates that both PKC $\alpha$ and DGK $\zeta$ can organize themselves into a local DAG-modulated signaling complex $[19,20]$. This DAG-based signalosome is instrumental in regulating the duration and amplitude of DAG in response to a variety of stimuli. Formation of this DAG signaling complex is the key to efficient DAG signaling transduction and localization to restricted subcellular sites. PKC $\alpha$ and DGK $\zeta$ are thought to have opposing functions in this signaling cascade. PKC $\alpha$ acts as a positive regulator whereas DGK $\zeta$ act as a negative regulator of DAG signaling $[19,20]$.

Human heart failure is linked to increased PKC $\alpha$ activity [12]. This linkage is surprising, as PKC $\alpha$ activity in most cell types is exquisitely regulated $[19,20]$. PKC $\alpha$ activity in cardiomyocytes is also tightly regulated through a molecular braking mechanism. As soon as PKC $\alpha$ is activated, the molecular brakes in place to check this increase in PKC $\alpha$ concentration should also kick in, acting on the common activator. This molecular braking mechanism begs the question: why is PKC $\alpha$ activity increased and maintained during heart failure despite the concurrent activation of a braking mechanism? Here, I propose an explanation based on data analyzing a local DAG signaling cascade that may be responsible for the increase in PKC $\alpha$ activity during HF.

The purpose of this study is to investigate how positive and negative effector molecules interact with each other during G $\alpha$ q signaling in the context of angiotensin II-induced heart failure. The rationale for this study is derived from the following experimental observations: (1) Cardiac-specific overexpression

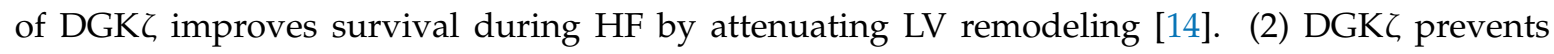
translocation of PKC $\alpha$ from the cytosol to the plasma membrane in response to angiotensin-II induced activation $[13,14]$. (3) DGK $\zeta$ and PKC $\alpha$ can organize themselves into a local signaling complex $[19,20]$. (4) PKC $\alpha$ overexpression negatively affects contractility [9-12]. (5) PKC $\alpha$ down-regulation prevents fibrosis and subsequent alteration of cardiac structure [11-14]. (6) PKC $\alpha$ is activated in a precise spatiotemporal manner $[19,20]$. (7) The activation of PKC $\alpha$ depends on the availability of DAG, 
a tightly-regulated signaling lipid $[10,19,20]$. (8) A local DGK - -PKC $\alpha$ signaling complex terminates DAG signaling by converting DAG to phosphatidic acid (PA). PA, in turn, negatively regulates PKC $\alpha$ activity $[19,20]$. (9) PKC $\alpha$ can induce its own activation by phosphorylating and deactivating DGK [21]. These observations suggest that DAG signaling in cardiomyocytes is regulated through a complex regulatory structure.

I propose DAG signaling homeostasis during Ang II-induced heart failure can be regulated through a molecular loop between the positive and negative effector molecules PKC $\alpha$ and DGK This proposed molecular loop may improve understanding of the molecular mechanisms involved in the complex spatiotemporal organization of DAG-PKC $\alpha-\mathrm{DGK} \zeta$ signaling during post-MI cardiac remodeling events. Using a computational model, this study shows the proposed molecular loop has a dual regulatory character. During basal conditions, a net negative feedback loop may prevail and regulate local DAG concentration. Interestingly, upon stimulation conditions, a positive feedback effect on DAG signaling is observed. This positive feedback can possibly explain the link between persistently high DAG levels and malignant outcomes. The transition from negative to positive feedback depends on the local biosynthesis rate of DAG and, in turn, on the mutual interactions between positive and negative DAG effector molecules.

The results of this study imply that a complex sequence of molecular events may regulate DAG homeostasis in a precise, spatiotemporal manner. This regulation includes DAG-induced translocation of PKC $\alpha$ and DGK $\zeta$ from the cytosol to the plasma membrane, formation of a local signaling complex between PKC $\alpha$ and DGK $\zeta$, and conversion of DAG to PA through the action of this signaling complex. Then, the complex is de-stabilized and PKC $\alpha$ and DGK , return to the cytosol. At the molecular level, these complex events generate a net negative feedback effect on DAG signaling. My computational model indicates that, after MI, DAG homeostasis is likely disrupted due to a positive feedback signal. My model shows the origin of a post-MI positive feedback loop is complex. It is likely modulated through a sequence of molecular events including DAG-induced activation of $\mathrm{PKC} \alpha$, dissociation of the PKC $\alpha-\mathrm{DGK} \zeta$ signaling complex, and PKC $\alpha$ mediated phosphorylation of DGK $\zeta$. After these events, it includes translocation of active PKC $\alpha$ from the cell membrane to the cytosol, and deactivation of active PKC $\alpha$ in the cytosol.

\section{Results}

\subsection{Local DAG Signaling Regulation in Cardiomyocytes}

The model I propose in Figure 1 describes local regulation of DAG homeostasis in cardiomyocytes. The model is composed of two molecular components: 1 . PKC $\alpha$, the target protein of DAG signaling, can exist in one of four states: cytosolic dormant $\left(\mathrm{PKC}_{\mathrm{II}} \alpha\right)$, inactive membrane $\left(\mathrm{PKC}_{\mathrm{I}} \alpha\right)$, active membrane

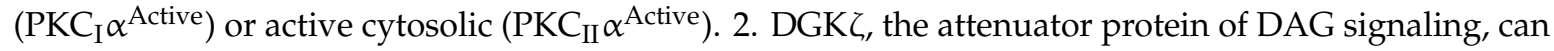
be in one of three states: cytosolic $\left(\mathrm{DGK}_{\mathrm{II}} \zeta\right)$, active membrane $\left(\mathrm{DGK}_{\mathrm{I}} \zeta\right)$, or phosphorylated/inactive membrane $\left(\mathrm{DGK}_{\mathrm{I}} \zeta_{\mathrm{P}}\right)$. Both these components migrate to the plasma membrane in a DAG-dependent manner. Once in the plasma membrane, DGK $\zeta$ forms complex $C_{1}$ with PKC $\alpha$. These two components interact in a closed loop and regulate DAG homeostasis in a negative feedback loop. Here, I assume that, during pathological conditions, complex $C_{1}$ is activated by DAG binding to PKC $\alpha$. Once active, $\mathrm{C}_{1}$ becomes unstable and dissociates into its components $\mathrm{DGK}_{\mathrm{I}} \zeta$ and $\mathrm{PKC}_{\mathrm{I}} \alpha^{\text {Active }}$. In turn, active $\mathrm{PKC}_{\mathrm{I}} \alpha^{\text {Active }}$ phosphorylates and deactivates DGK $\zeta$. My model assumes that, once active, PKC $\alpha$ can stimulate its own activation through a persistent positive feedback loop. This positive feedback may be linked to heart failure [8-15]. 
a

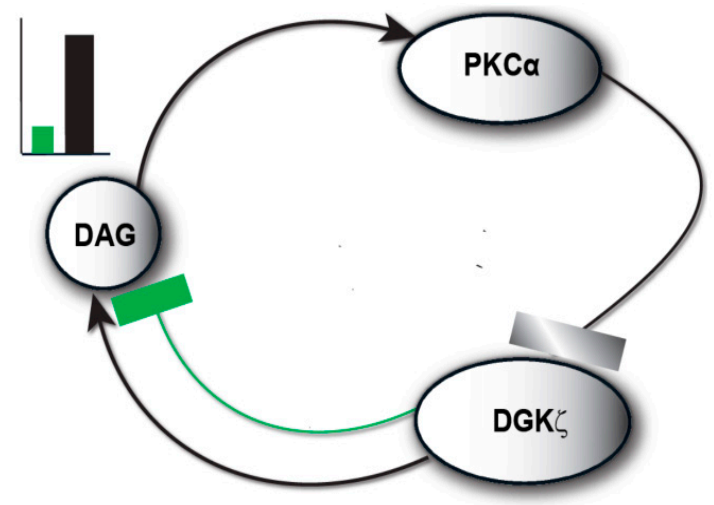

b

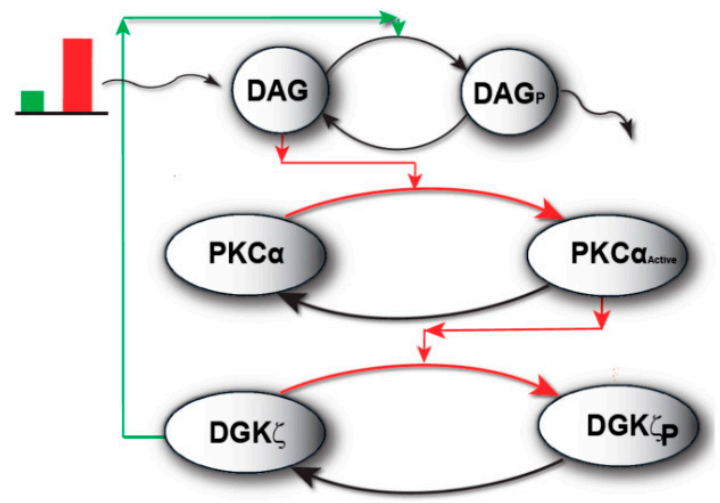

Figure 1. The regulatory molecular loop between the target protein kinase $C \alpha(\mathrm{PKC} \alpha)$ and attenuator

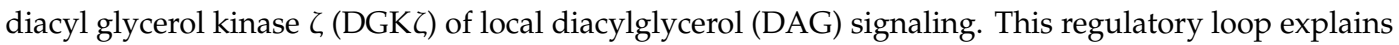
how the second messenger, DAG, is tightly and dynamically regulated through the formation of a DAG-induced DGK - -PKC $\alpha$ signaling complex. (a) Conceptual diagram of a DAG regulatory loop. This wiring diagram shows that during stimulation levels (green pulse) of low intensity (normal physiological state), DAG homeostasis is regulated through a negative feedback signal. However, at higher levels of stimulation (black pulse; possible pathological state), DAG homeostasis is disrupted due to a positive feedback effect. The positive feedback on DAG signaling is due to DAG-induced activation of PKC $\alpha$ which, in turn, leads to dissociation of signaling complex and subsequent deactivation of DGK $\zeta$ through phosphorylation. (b) Detailed description of the DAG regulatory loop. Here, during normal state (green pulse and green feedback line), the active DGK $\zeta$ molecule phosphorylates DAG, thereby preventing the PKC $\alpha$ activation. On higher levels of stimulation (red pulse and red feedback line), the local DAG levels increase so sharply that it outpaces the metabolizing ability of the DGK $\zeta-\mathrm{PKC} \alpha$ signaling complex. Thus, net local accumulation of DAG activates PKC $\alpha$, causing the dissociation of the signaling complex. The active PKC $\alpha$, in turn, phosphorylates and deactivates DGK $\zeta$. This loop also shows that, once active, the PKC $\alpha$ molecule can stimulate its own activation through persistent positive feedback, which can be linked with blunted contractility in heart failure.

The model of local DAG signaling (Figure 2) assumes that PKC $\alpha$ can reside in four distinct states. The first is an inactive, dormant state that cannot phosphorylate substrates and resides only in the cytosol. Second, PKC $\alpha$ may reside in an inactive state that cannot phosphorylate substrates and resides only in the plasma membrane. Third, PKC $\alpha$ may exist in an active state obtained when inactive PKC $\alpha$ binds to DAG at the plasma membrane compartment. This active state can phosphorylate other substrates including $\mathrm{DGK}_{\mathrm{I}} \zeta$. Last, PKC $\alpha$ may exist in an active state in the cytosol. This active state is obtained when active PKC $\alpha$ translocates from the plasma membrane back to the cytosol [11,22]. This form of PKC $\alpha$ is prone to degradation. If PKC $\alpha$ does not degrade, it becomes deactivated, and exists as the dormant form of $\alpha$-enzyme. This active state can also phosphorylate other substrates. The faithful translocation, activation, and re-translocation/de-activation model of PKC $\alpha$ may be more 
complex than the four-state model assumed here [11]. A more complex model may consider the exact details of the molecular events involved when PKC $\alpha$ translocates from the cytosol to the plasma membrane. The model must also take into account the inhibition of PKC $\alpha$ translocation by DGK $\zeta$, as well as enzyme anchoring at the plasma membrane, re-translocation of active PKC $\alpha$ to the cytosol, PKC $\alpha$ degradation, and deactivation. These processes are complex but have been approximated within our model by simpler biochemical kinetic events. These simplified kinetic events preserve key qualitative features of the biochemical interactions within the model. In the computational simulations, PKC $\alpha$ translocation from the cytosol to the plasma membrane is assumed to be regulated through a kinetic event that is directly proportional to the DAG concentration at the plasma membrane. It is assumed the DAG concentration is inversely proportional to the concentration of DGK $\zeta$. I also assumed there were non-negligible basal levels of dormant cytosolic PKC $\alpha(10 \mathrm{pg} / \mathrm{mL})$ and DGK $(10 \mathrm{pg} / \mathrm{mL})$. In contrast, the basal concentration of all other forms of PKC $\alpha$ and DGK $\zeta$ are assumed to be negligibly small. The biochemical reaction rates for the DGK $\zeta-P K C \alpha$ molecular loop have been published in previous work [19-21]. In order to determine the degradation rates of PKC $\alpha$, constants with experimentally measured rates were used [22].

DGK $\zeta$ inhibits DAG translocation in my proposed model. Upon stimulation, local generation of DAG induces both PKC $\alpha$ and DGK $\zeta$ translocation. Once in the plasma membrane, both the DAG signaling target and attenuator proteins form biochemical complex $C_{1}$. This complex, in turn, stimulates the degradation of second messenger DAG, thus restoring homeostasis. In the proposed model, the rate of DAG generation is counterbalanced by a negative feedback effect generated through $C_{1}$-mediated DAG phosphorylation. Under normal physiological conditions, DAG concentration is regulated in this tight spatiotemporal manner.

The model assumes that during pathological conditions the local rate of DAG generation far exceeds the rate of $C_{1}$-mediated removal. This dynamic leads to a transient increase in local DAG concentration. Local accumulation of DAG, in turn, activates $C_{1}$ through binding PKC $\alpha$. Once active, $C_{1}$ dissociates into $\mathrm{PKC}_{\mathrm{I}} \alpha^{\text {Active }}$ and $\mathrm{DGK}_{\mathrm{I}} \zeta$. Active $\mathrm{PKC}_{\mathrm{I}} \alpha^{\text {Active }}$ deactivates $\mathrm{DGK}_{\mathrm{I}} \zeta$ through phosphorylation. In this model, $\mathrm{PKC} \alpha$ stimulates its own activation through a persistent positive feedback loop. Prolonged PKC $\alpha$ activation may be linked to systolic and diastolic dysfunction. Although the model represents a simplified version of $\mathrm{G} \alpha \mathrm{q} / \mathrm{DAG}$ signaling, it captures the essential features of local DAG signaling in cardiomyocytes. This local DAG signaling loop is described by a set of biochemical reactions (Equations (1)-(16)).

Local DAG generation in response to angiotensin II-mediated activation of the GPCR pathway is a complex regulatory process [7,9]. GPCR agonist angiotensin II stimulates phospholipase C-mediated hydrolysis of phosphatidylinositol 4,5-biphosphate to produce inositol triphosphate and DAG [7]. Although this study primarily focuses on the downstream signaling of the angiotensin II-stimulated GPCR pathway, Local DAG biosynthesis is modeled using a brief stimulatory pulse that varies in intensity. This pulse mimics the effects of GPCR agonist angiotensin II. This simple approach ignores the finer details of angiotensin II-induced DAG biosynthesis [7]. As the primary focus of this study is how DAG homeostasis is regulated, not DAG biosynthesis, the simple approach was chosen so as not to obscure any overarching results with fine details. 


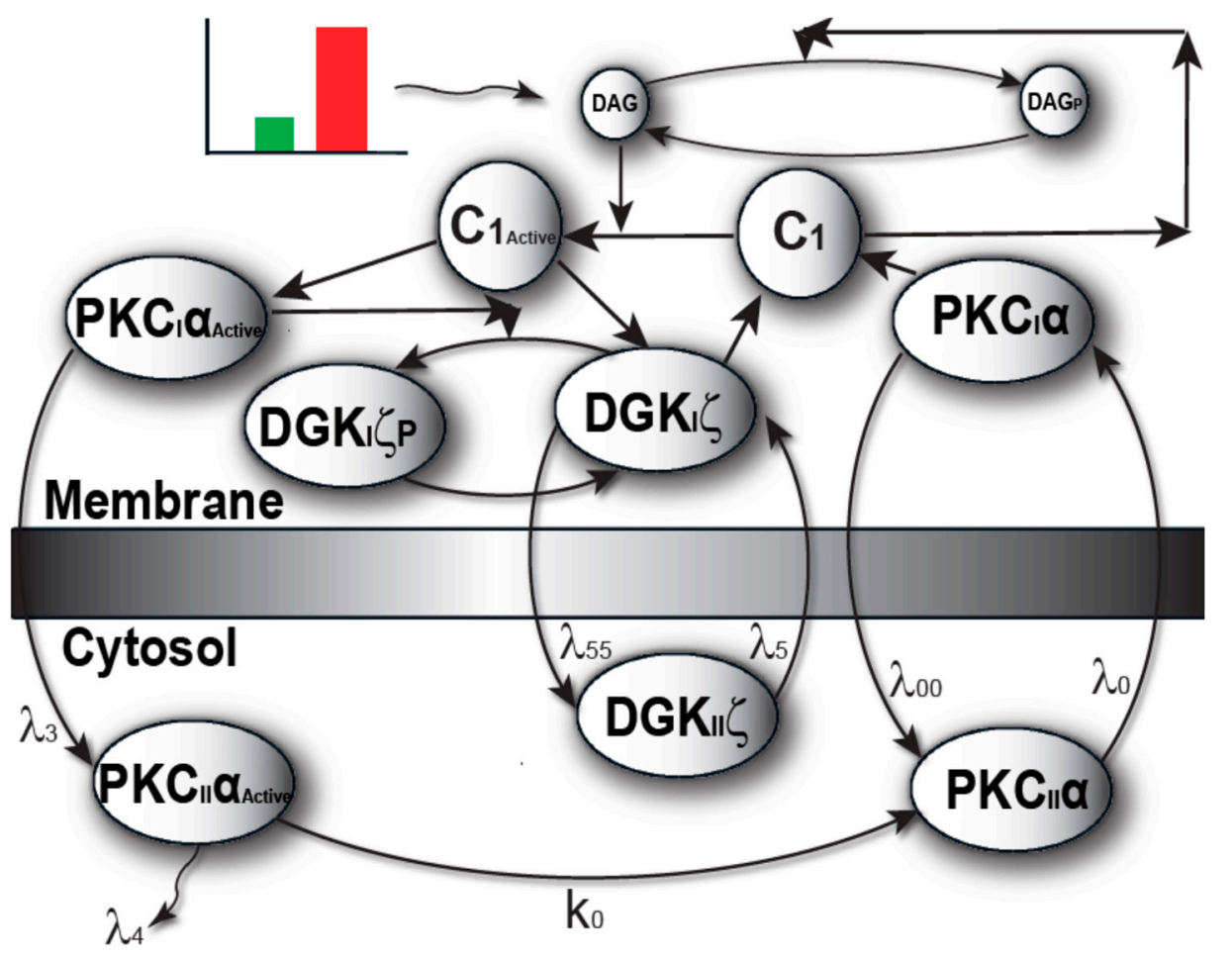

Figure 2. The two-compartment regulatory model of local DAG signaling in cardiomyocytes. Here, one of the compartments is cytosol, whereas the other compartment is plasma membrane. This model explains the spatial and temporal regulation of DAG-PKC $\alpha-\mathrm{DGK} \zeta$ signaling in G $\alpha$-induced heart failure. This model describes a sequence of complex events involving the translocation, activation, association/dissociation, phosphorylation/deactivation and redistribution of target and attenuator molecules of DAG signaling in cardiomyocytes. Here, the PKC $\alpha$ molecule (DAG target) exists in four states: (1) dormant PKC $\alpha$ enzyme residing in cytosol $\left(\mathrm{PKC}_{\mathrm{II}} \alpha\right) ;(2)$ active $\mathrm{PKC} \alpha$ enzyme residing in cytosol ( $\mathrm{PKC}_{\mathrm{II}} \alpha^{\text {Active }}$ ); (3) inactive molecule residing in the plasma membrane ( $\left.\mathrm{PKC}_{\mathrm{I}} \alpha\right)$; (4) active molecule residing in the plasma membrane ( $\left.\mathrm{PKC}_{\mathrm{I}} \alpha^{\text {Active }}\right)$. Similarly, the attenuator of DAG signaling,

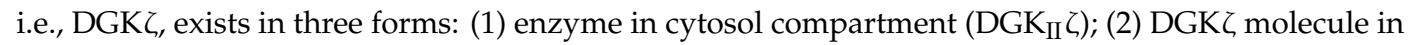
the plasma membrane (DGK $\zeta$ ); (3) phosphorylated and inactive molecule residing in the membrane compartment $\left(\mathrm{DGK}_{\mathrm{I}} \zeta_{\mathrm{P}}\right)$. During the normal conditions (green pulse), when DAG levels are low, both $\mathrm{PKC}_{\mathrm{II}} \alpha$ and $\mathrm{DGK}_{\mathrm{II}} \zeta$ migrate to the plasma membrane in a DAG-dependent manner. Once at the plasma membrane they form a complex $C_{1}$, thus, metabolizing the DAG through phosphorylation. On high intensity stimulation (red pulse), higher levels of DAG are generated, thus stimulating the migration of larger quantities of PKC $\alpha$ and DGK $\zeta$ from cytosol to membrane. In this case, the local DAG levels increase so sharply that the ability of complex $C_{1}$ to metabolize DAG to PA is outpaced by generation, leading to a transient increase in local concentrations of the second messenger. In turn, this leads to the activation of complex $C_{1}$ through the DAG binding with PKC $\alpha$. Upon activation, the complex $\mathrm{C}_{1}{ }^{\mathrm{A}}$ becomes unstable and dissociates into $\mathrm{PKC}_{\mathrm{I}} \alpha^{\text {Active }}$ and $\mathrm{DGK}_{\mathrm{I}} \zeta$. The active enzyme $\mathrm{PKC}_{\mathrm{I}} \alpha^{\mathrm{A}}$ phosphorylates and deactivates the $\mathrm{DGK}_{\mathrm{I}} \zeta$ molecule. The active molecule $\mathrm{PKC}_{\mathrm{I}} \alpha^{\text {Active }}$ migrates back to cytosol. Once in cytosol, the active form of PKC $\alpha$ enzyme could either degrade or deactivates into its dormant form and is stored for the next translocation-activation-re-translocation cycle. Through a complex process, the inactive $\mathrm{DGK}_{\mathrm{I}} \zeta_{\mathrm{P}}$ molecule is dephosphorylated, thus, again, forming a complex $C_{1}$, and eventually reducing the increase in DAG levels and restoring its homeostasis in the plasma membrane.

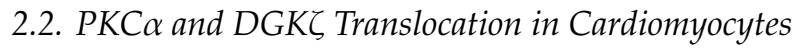

Using the computation model, I next determined the translocation characteristics of both the target and attenuator molecules of DAG signaling in cardiomyocytes. In the model, translocation characteristics were determined by measuring the membrane-to-cytosol $(\mathrm{M} / \mathrm{C})$ ratio of target and 
attenuator molecules. The $\mathrm{M} / \mathrm{C}$ ratio describes the relative distribution of $\mathrm{PKC} \alpha$ and $\mathrm{DGK} \zeta$ molecules in the membrane and cytosolic compartments. For PKC $\alpha$, this ratio is an indirect index of activation. According to the proposed model, the $\mathrm{M} / \mathrm{C}$ ratio for $\mathrm{DGK} \zeta$ could be indirectly linked to kinase deactivation. A higher $\mathrm{M} / \mathrm{C}$ ratio indicates high rates of molecular migration from the cytosol to the plasma membrane. Ang II-induced biosynthesis of DAG was implemented using a three-minute pulse, as described in the previous section. The strength of the pulse is described by an arbitrary parameter $S_{1}$. Here, the parameter $S_{1}$ is set at three arbitrary levels: $0.5,2$ and 6 . In the absence of a pulse (Figure 3 and Figure S1, solid lines) there is no de novo DAG biosynthesis and the system is fixed in its basal state. In the basal state, both molecules reside in the cytosol with no possibility of translocation.
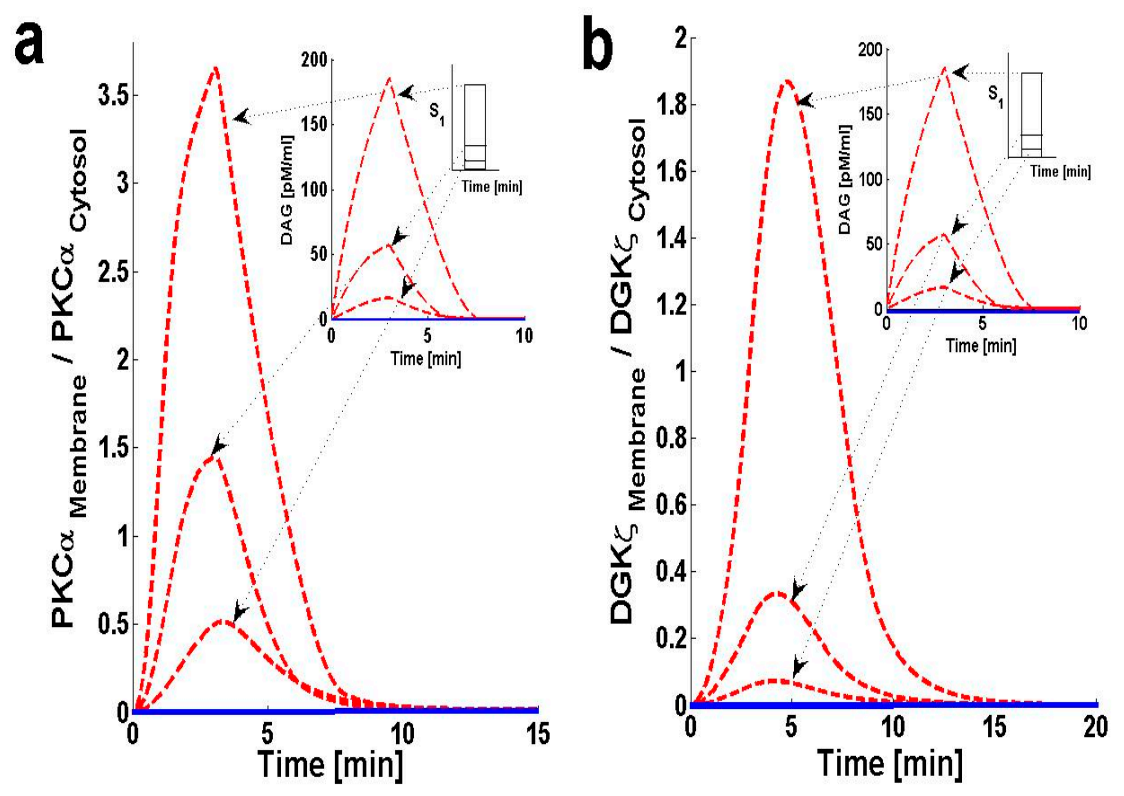

Figure 3. Membrane-to-cytosol $(\mathrm{M} / \mathrm{C})$ ratios of target and attenuator molecules of DAG signaling. These results show that pulse-like (duration of $3 \mathrm{~min}$ and three different levels of pulse strength are used i.e., " $\mathrm{S}_{1}$ " $=0.5,2$ and 6) stimulation leads to the rapid generation of DAG. The generation of second messenger, in turn, stimulates the translocation of both the target and attenuator molecules of DAG signaling from cytosol to membrane. Here, the solid line represents the non-stimulation condition, whereas the dashed line represents stimulation. (a) $\mathrm{M} / \mathrm{C}$ ratios of $\mathrm{PKC} \alpha$ with respect to different levels of pulse-like stimulation mimicking the GPCR agonist angiotensin II (Ang. II). These results show that Ang. II like stimulation leads to rapid de-novo generation of DAG, which, in turn, stimulates the translocation of both PKC $\alpha$ and DGK , from cytosol to membrane. Here, the translocation rates are set as linear functions of DAG concentration. At low stimulation levels only a small amount of DAG is generated at the plasma membrane thus, inducing the migration of only a small pool of PKC $\alpha$ to the membrane. At the membrane, PKC $\alpha$ forms a biochemical complex $C_{1}$ with DGK $\zeta$ and stimulates the DAG conversion to PA. Once DAG homeostasis is restored, the complex $\mathrm{C}_{1}$ decomposes into DGK $\zeta$ and $\mathrm{PKC}_{\mathrm{I}} \alpha$ molecules; these, in turn, quickly re-translocate to the cytosol compartment. At higher stimulation levels, a much larger quantity of DAG is generated, thus stimulating the translocation of a much larger pool of PKC $\alpha$ from cytosol to membrane. High-intensity stimulation leads to a much larger $\mathrm{M} / \mathrm{C}$ ratio of PKC $\alpha$ and enhanced residence time in membrane compartment. (b) $\mathrm{M} / \mathrm{C}$ ratios of DGK $\zeta$ with respect to different levels of stimulation. The DGK $\zeta \mathrm{M} / \mathrm{C}$ ratio rapidly increases due to a sharp increase in DAG concentration on stimulation. Here, the translocation event of DGK $\zeta$ is also modeled as a linear proportional relationship to the free concentration of DAG.

In the presence of a pulse, the $\mathrm{M} / \mathrm{C}$ ratios of both PKC $\alpha$ (Figure 3a, dashed line) and DGK $\zeta$ (Figure 3b, dashed line) increase to their maximum levels followed by gradual clearance of signal. The temporal dynamics depicted in Figure 3 clearly show two phases of translocation. The first is an early phase in which the target and attenuator molecules of DAG signaling migrate from the cytosol to 
the plasma membrane. The first phase is followed by a second phase where both molecules translocate back to the cytosol. These results indicate that the migration of PKC $\alpha$ and DGK , from the cytosol to the plasma membrane depends on local DAG concentration at the plasma membrane. It is also linked to the de novo biosynthesis rate of DAG, which is controlled throughout the duration. It is also linked to the amplitude of pulse stimulation at the plasma membrane (Figure 3a,b, inset; pulse strength parameter $\mathrm{S}_{1}=0.5,2$ and 6 ).

Translocation of PKC $\alpha$ from the cytosol to the plasma membrane is controlled by two key parameters. The first parameter is DAG concentration at the plasma membrane. Here, the PKC $\alpha$ translocation rate has been set to be directly proportional to DAG concentration (Figure S4 and Table S1). The second parameter is $\mathrm{DGK}_{\mathrm{II}} \zeta$ concentration in the cytosol. Here, the PKC $\alpha$ translocation rate is indirectly dependent on $\mathrm{DGK}_{\mathrm{II}} \zeta$ concentration in the cytosol, as $\mathrm{DGK}_{\mathrm{II}} \zeta$ concentration controls DAG levels at the plasma membrane. In the simulation, when $S_{1}$ is set at low levels $\left[S_{1}=0.5,2\right]$ a small amount of DAG is generated at the plasma membrane. This induces the migration of a small pool of PKC $\alpha$ to the membrane. PKC $\alpha$ forms complex $C_{1}$ with DGK $\zeta$ in the membrane compartment and induces the metabolism of DAG to PA. This process restores DAG homeostasis. Once DAG homeostasis is restored, complex $\mathrm{C}_{1}$ decomposes into $\mathrm{PKC}_{\mathrm{I}} \alpha$ and $\mathrm{DGK}_{\mathrm{I}} \zeta$. $\mathrm{PKC}_{\mathrm{I}} \alpha$ and $\mathrm{DGK}_{\mathrm{I}} \zeta$, in turn, quickly translocate back to the cytosol without undergoing complete activation and deactivation cycles, respectively. In contrast, during high-intensity stimulation $\left[S_{1}=6\right]$ a large quantity of DAG is generated. This stimulates translocation of a large pool of $\mathrm{PKC} \alpha$ from the cytosol to the plasma membrane. High levels of stimulation lead to larger M/C ratios for PKC $\alpha$ (Figure 3a).

The translocation pattern of DGK $\zeta$ (Figure 3b, dashed line) is identical to that of PKC $\alpha$. Upon stimulation, the $\mathrm{M} / \mathrm{C}$ ratio rapidly increases due to a sharp increase in $\mathrm{DAG}$ concentration. Translocation

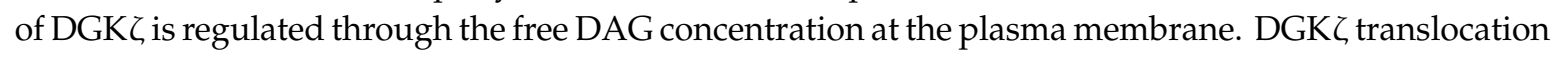
is modeled as a proportional function of free DAG concentration. These results also indicate that high levels of stimulation lead to a larger M/C ratio for DGK $\zeta$ (Figure 3b). These simulations also show that, for $S_{1}=[0.5,2$, and 6$]$, the maximum value of the M/C ratio for PKC $\alpha$ is always larger than the M/C ratio for DGK $\zeta$. This is due to the PKC $\alpha$ translocation rate's dependence on DAG concentration.

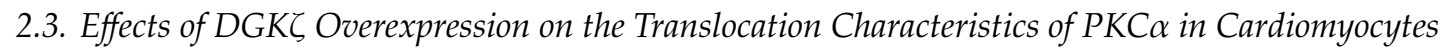

The chance of survival after MI increases upon cardiac-specific overexpression of DGK $\zeta$. This is due to the attenuation of post-infarction LV remodeling. The survival rate almost doubles with DGKC overexpression [14]. Observations in transgenic mice indicate that, independent of hemodynamic

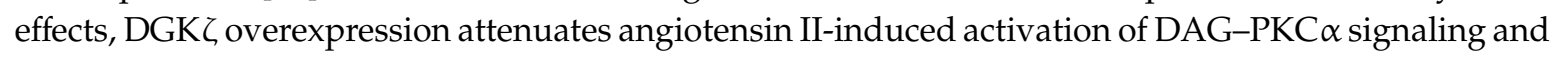
subsequent contractile dysfunction [13-15]. Experimental observations show that, in wild type mouse hearts, angiotensin II induces the translocation of PKC $\alpha$ from the plasma membrane to the cytosol [13]. This translocation event is blocked by DGK $\zeta$ overexpression [13,15]. I set out to address the question of why translocation is blocked by DGK $\zeta$ overexpression by using a simple regulatory model for local DAG signaling. In this model's simulations, DGK $\zeta$ overexpression was implemented by adjusting the DGK $\zeta$-to-PKC $\alpha$ ratio under initial conditions. In the case where DGK $\zeta$ is not overexpressed, the DGK $\zeta$-to-PKC $\alpha$ ratio is set at 1 . For simulations mimicking 2 -fold and 9-fold overexpression over basal levels of DGK $\zeta$, the ratios are set at 2 and 9, respectively. Here, the effects of DGK $\zeta$ overexpression on migration of target and attenuator molecules of DAG signaling (Figure $4 a, b$ ) were tracked. Figure $4 a$ shows that overexpression of DGK $\zeta$ restricts PKC $\alpha$ translocation from the cytosol to the plasma membrane. At 2-fold DGK $\zeta$ overexpression, a significant reduction in the $\mathrm{M} / \mathrm{C}$ ratio of $\mathrm{PKC} \alpha$ is observed. For 9-fold overexpression, a further reduction in the $\mathrm{M} / \mathrm{C}$ ratio is observed and the $\mathrm{M} / \mathrm{C}$ ratio for this case is less than 1. At even higher levels of DGK $\zeta$ overexpression, PKC $\alpha$ translocation to the plasma membrane can be completely eliminated. These results also show that overexpression of DGK $\zeta$ only slightly influences the migration characteristics of attenuator molecules of DAG signaling (Figure $4 \mathrm{~b}$ ). These results indicate that the maximum value of the $\mathrm{M} / \mathrm{C}$ ratio of DGK $\zeta$ decreases from 1.84 to 0.78 as the overexpression level increases from basal levels to 9-fold overexpression. 

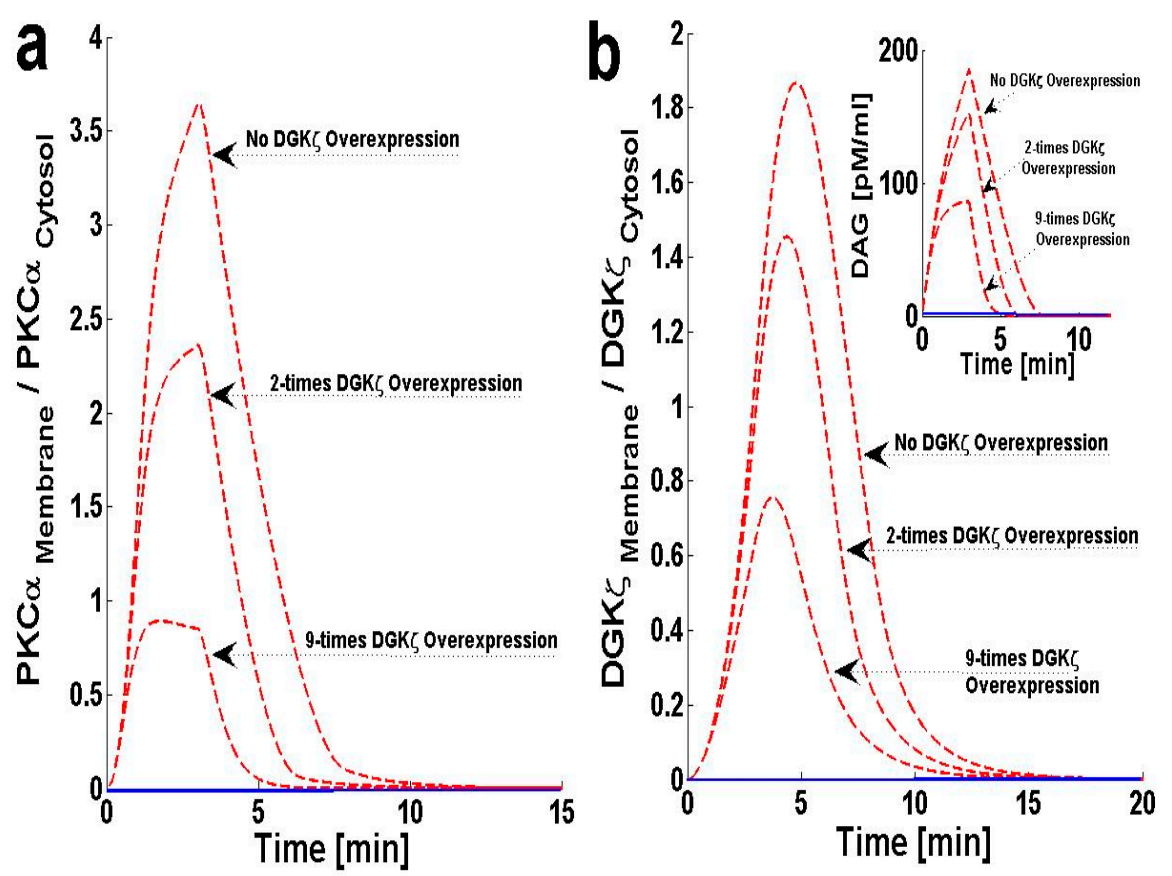

Figure 4. The effect of cardiac-specific overexpression of diacylglycerol kinase $\zeta$ (DGK $\zeta$ ) on the membrane-to-cytosol $(\mathrm{M} / \mathrm{C})$ ratio of target and attenuator molecules of DAG signaling in cardiomyocytes. For this case, the pulse intensity is set at level 6 for 3 min leading to rapid generation of DAG. The rapid generation of second messenger, in turn, stimulates the translocation of both the DAG target and attenuator molecules from cytosol to membrane. Here, the overexpression of DGK $\zeta$ is modeled through increasing the ratio of DGK $\zeta$ to PKC $\alpha$ in the initial conditions. For the no overexpression case, the DGK $\zeta$ to PKC $\alpha$ ratio is set at 1, whereas for simulations mimicking 2 -fold and 9-fold overexpression this ratio is set at 2 and 9 respectively. Here, the solid line represents the non-stimulation condition, whereas the dashed line represents stimulation. (a) M/C ratios of PKC $\alpha$ at different overexpression levels of DGK $\zeta$. Simulations are performed with no overexpression, 2-fold and 9-fold overexpression of DGK $\zeta$ molecule. These results show that overexpression of DGK $\zeta$ molecule restricts the PKC $\alpha$ translocation from cytosol to membrane. At almost 2-fold DGK $\zeta$ overexpression, a significant reduction in the $\mathrm{M} / \mathrm{C}$ ratio of $\mathrm{PKC} \alpha$ is observed. For 9 -fold overexpression, a further reduction in $\mathrm{M} / \mathrm{C}$ ratio is noticed and the $\mathrm{M} / \mathrm{C}$ ratio for this case is even lesser than 1 . At even higher DGK the PKC $\alpha$ translocation to membrane is completely eliminated (results not shown). (b) $\mathrm{M} / \mathrm{C}$ ratios of DGK $\zeta$ at different overexpression levels of DGK $\zeta$. The maximum value of $\mathrm{M} / \mathrm{C}$ ratio first decreases from 1.84 to 1.42 as the overexpression level increase from no overexpression to 2-fold. Interestingly, as the overexpression further increases to 9 -fold, the maximum value of $\mathrm{M} / \mathrm{C}$ ratio decreases to 0.78 .

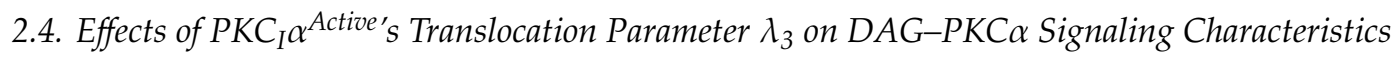

In this model, PKC $\alpha$ can cycle between four states: $\mathrm{PKC}_{\mathrm{II}} \alpha, \mathrm{PKC}_{\mathrm{I}} \alpha, \mathrm{PKC}_{\mathrm{I}} \alpha^{\text {Active }}$ and $\mathrm{PKC}_{\mathrm{II}} \alpha^{\mathrm{Active}}$. $\mathrm{PKC} \alpha$ cycling is regulated through processes like translocation, activation, translocation back to the previous location, and deactivation. The model assumes that once PKC $\alpha$ is activated in the membrane compartment, it must translocate back to the cytosol before it is deactivated and enters a dormant state. This assumption is based on several lines of experimental observation describing the activation and deactivation cycles of cPKCs $[11,23]$. From these experimental results, I propose that $\mathrm{PKC}_{\mathrm{I}} \alpha$ Active translocation back to the cytosol may influence DAG-PKC $\alpha$ signaling. In order to study this question, I blocked the translocation of $\mathrm{PKC}_{\mathrm{I}} \alpha^{\text {Active }}$ from the plasma membrane back to the cytosol by decreasing $\lambda_{3}$. The results indicate that, as $\lambda_{3}$ is blocked, the maximum value of the $\mathrm{M} / \mathrm{C}$ ratios for both PKC $\alpha$ and DGK $\zeta$ increase (Figure $5 \mathrm{a}, \mathrm{b}$ ).

Figure $5 b$, inset, shows that blocking this translocation back to the cytosol not only increases the maximum DAG concentration, but also prolongs the duration during which the concentration is 
non-negligible. These results indicate that inhibition of $\mathrm{PKC}_{\mathrm{I}} \alpha^{\text {Active }}$ translocation back to the cytosol reduces the ability of complex $C_{1}$ to metabolize DAG, as PKC $\alpha$ is possibly trapped in the $\mathrm{PKC}_{\mathrm{I}} \alpha^{\text {Active }}$ state in the plasma membrane.
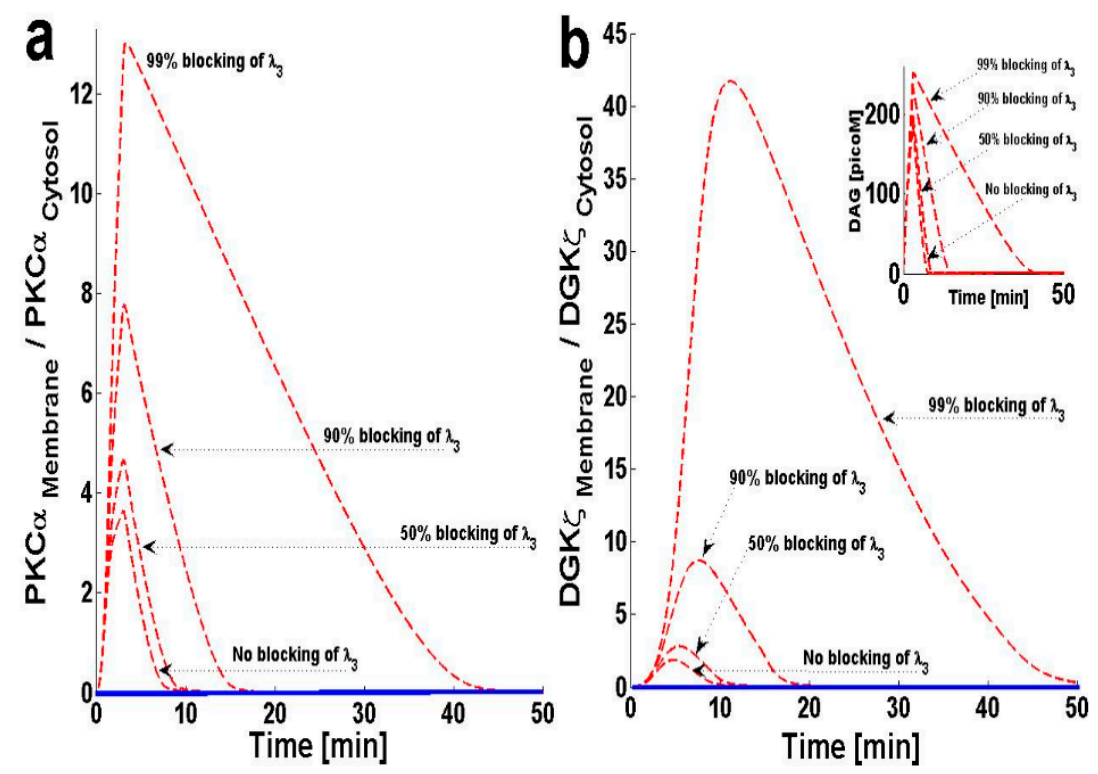

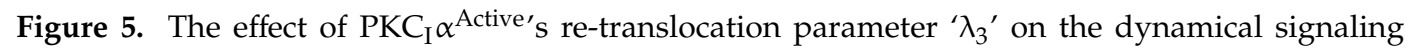
characteristics of the DAG-PKC $\alpha-\mathrm{DGK} \zeta$ cascade. For these simulations, the intensity of pulse stimulation is set at 6 with a duration of $3 \mathrm{~min}$, leading to rapid generation of DAG. The rapid generation of second messenger, in turn, stimulates the translocation of both the DAG target and attenuator molecules from cytosol to membrane. Here, the solid line represents the non-stimulation condition, whereas the dashed line represents stimulation. (a) M/C ratios of PKC $\alpha$ with respect to the re-translocation parameter of active membrane-bound PKC $\alpha$ enzyme i.e., $\lambda_{3}$. These results show that as the re-translocation parameter is decreased the maximum value of $\mathrm{M} / \mathrm{C}$ ratio increases, and the duration for which it is non-negligible is also almost doubled. (b) M/C ratios of DGK $\zeta$ at different inhibition levels of $\lambda_{3}$. Inhibiting the re-translocation parameter of $\mathrm{PKC}_{\mathrm{I}} \alpha^{\text {Active }}$ from membrane to cytosol also increased the $\mathrm{M} / \mathrm{C}$ ratio of $\mathrm{DGK} \zeta$, and the duration for which it is non-negligible is prolonged (b-Inset) Temporal dynamics of "DAG" with respect to blocking of parameter $\lambda_{3}$. These results show that blocking $\lambda_{3}$ not only increased the maximum level of DAG but also the duration for which it is non-negligible.

\subsection{Effects of $P K C_{I I} \alpha^{A c t i v e ' s ~ D e a c t i v a t i o n ~ P a r a m e t e r ~} k_{0}$ on DAG-PKC $\alpha$ Signaling Characteristics}

As mentioned in the previous section, the PKC $\alpha$ cycle is regulated through four key processes: translocation, activation, re-translocation and deactivation. Similar to the re-translocation process, the deactivation process can also critically influence the PKC $\alpha$ cycle. Several lines of experimental observation show that, in the cytosol, $\mathrm{cPKC}$ is deactivated back to its dormant state through proteins like HSP60. In the next simulation, the effects of the $\mathrm{PKC}_{\mathrm{II}} \alpha^{\text {Active }}$ deactivation parameter, $\mathrm{k}_{0}$, were tested on DAG-PKC $\alpha$ signaling. The results show that much higher levels of deactivation parameter $\mathrm{k}_{0}$ are needed to effectively reduce the $\mathrm{M} / \mathrm{C}$ ratio of $\mathrm{PKC} \alpha$ (Figure 6a). These high levels thus restrict PKC $\alpha$ translocation from the cytosol to the membrane. At very high levels (99\% blocking) the maximum $\mathrm{M} / \mathrm{C}$ ratio decreases to 2.42 (Figure $6 \mathrm{a}$ ). This reduction in $\mathrm{M} / \mathrm{C}$ ratio is due to the disruption in the PKC $\alpha$ cycle associated with inhibition of deactivation parameter $k_{0}$. In contrast, the $M / C$ ratio of DGK $\zeta$ (Figure $6 \mathrm{~b}$ ) slightly increases with $\mathrm{k}_{0}$ blocking. The temporal dynamics of DAG are also affected (Figure $6 \mathrm{~b}$, inset) at different levels of $\mathrm{k}_{0}$ inhibition. These results show that significant blocking $(0-90 \%)$ of the deactivation parameter " $\mathrm{k}_{0}$ " does not lead to a significant increase in 'DAG' levels. Even $99 \%$ blocking of the deactivation parameter " $\mathrm{k}_{0}$ " only leads to a slight increase in the maximum levels of 'DAG' and a slight increase in the duration during which DAG levels are non-negligible. These results 
point towards the interesting possibility that " $\mathrm{k}_{0}$ " inhibition may serve as a strategy when designing therapeutic targets related to PKC $\alpha$ during heart failure.
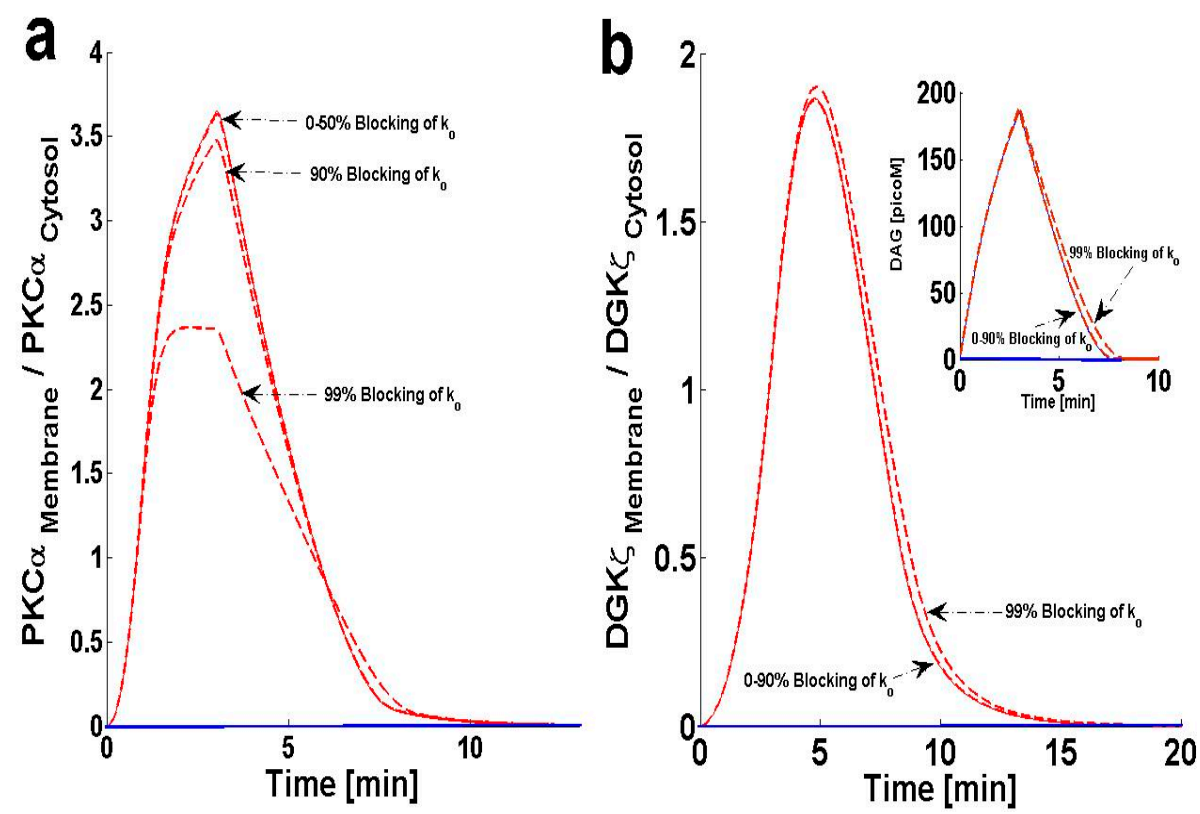

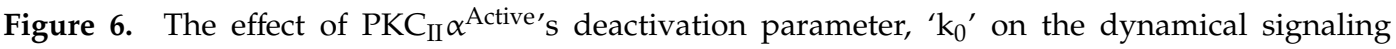
characteristics of the DAG-PKC $\alpha-\mathrm{DGK} \zeta$ molecular loop. For these simulations, the pulse intensity is set at 6 for $3 \mathrm{~min}$, leading to rapid generation of DAG. The rapid generation of second messenger, in turn, stimulates the translocation of both the DAG target and attenuator molecules from cytosol to membrane. Here, the solid line represents the non-stimulation condition, whereas the dashed line represents stimulation. (a) $\mathrm{M} / \mathrm{C}$ ratios of PKC $\alpha$ at different levels of ' $\mathrm{k}_{0}$ ' inhibition. These results show that blocking the deactivation parameter $\mathrm{k}_{0}$ effectively reduces the $\mathrm{M} / \mathrm{C}$ ratio of $\mathrm{PKC} \alpha$, thus restricting the translocation of PKC $\alpha$ from cytosol to membrane. At much higher blocking levels (99\% blocking), the $\mathrm{M} / \mathrm{C}$ ratio decreases to 2.42 . This reduction in $\mathrm{M} / \mathrm{C}$ ratio is due to the disruption in PKC $\alpha$ cycle which is caused by the inhibition of deactivation parameter $k_{0}$. (b) $M / C$ ratios of DGK $\zeta$ at different levels of ' $k_{0}$ ' inhibition. ( $b$, inset) temporal dynamics of "DAG" at different $k_{0}$ inhibition levels. These results show that extensive blocking $(0-90 \%)$ of the deactivation parameter " $\mathrm{k}_{0}$ " did not lead to a significant increase in 'DAG' levels. Even $99 \%$ blocking of the deactivation parameter " $\mathrm{k}_{0}$ " only leads to a slight increase in the maximum levels of 'DAG' and a slight increase in the duration for which it is non-negligible. These results provide an interesting possibility for " $\mathrm{k}_{0}$ " inhibition as a potential therapeutic target for PKC $\alpha$ in heart failure.

\subsection{Effect of Forward Rate Constant, ' $k_{2}$ ', on DAG-PKC $\alpha$ Signaling Characteristics}

The proposed model suggests that formation of complex $C_{1}$ is crucial for regulating DAG homeostasis at the plasma membrane. Here, I investigate how the kinetics of complex $C_{1}$ formation influence DAG-PKC $\alpha$ signaling. In order to investigate this question, two sets of computational experiments were conducted. The first experimental set involves increasing the forward rate constant (Figure 7), whereas the second set of simulations are focused on blocking the $k_{2}$ parameter (Figure S3), or the rate constant for complex $C_{1}$. The results of this study show that increasing the parameter $\mathrm{k}_{2}$ effectively decreases the $\mathrm{M} / \mathrm{C}$ ratio of $\mathrm{PKC} \alpha$, and also reduces the duration for which the ratio is non-negligible (Figure 7a). These data also show that blocking the parameter $\mathrm{k}_{2}$ effectively increases the $\mathrm{M} / \mathrm{C}$ ratio of $\mathrm{PKC} \alpha$ and prolongs the duration for which it is non-negligible (Figure S3a). The results for the M/C ratio of DGK $\zeta$ (Figure $7 \mathrm{~b}$ and Figure S3b) also follow the same pattern. 

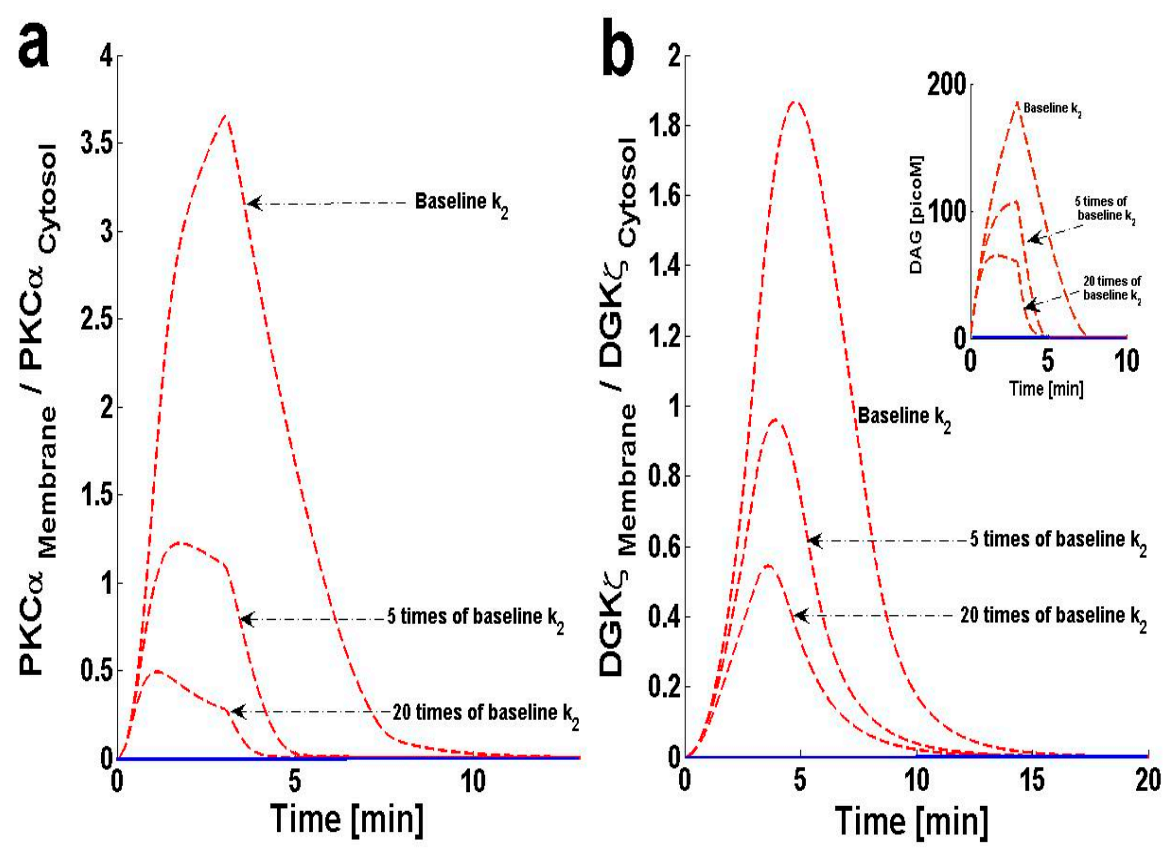

Figure 7. The effect of increasing the forward rate constant, ' $\mathrm{k}_{2}{ }^{\prime}$ on the dynamical signaling characteristics of the DAG-PKC $\alpha-D G K \zeta$ signaling complex. The parameter ' $k_{2}{ }^{\prime}$ represents the formation rate constant during the interaction of $\mathrm{PKC}_{\mathrm{I}} \alpha$ and DGK $\zeta$ at plasma membrane to form the biochemical complex $C_{1}$. For these simulations, the pulse intensity is set at 6 for 3 min leading to rapid generation of DAG. The rapid generation of second messenger in turn stimulates the translocation of both the DAG target and attenuator molecules from cytosol to membrane. Here, the solid line represents the basal condition, whereas the dashed line represents stimulation. (a) M/C ratios of PKC $\alpha$ at different increasing levels of ' $k_{2}$. These results show that increasing the formation rate constant $k_{2}$ effectively reduces the $\mathrm{M} / \mathrm{C}$ ratio of $\mathrm{PKC} \alpha$, and also the duration for which it is non-negligible. (b) M/C ratios of DGK $\zeta$ at different increasing levels of ' $k_{2}$ '. (b-Inset) Temporal dynamics of "DAG" with respect to increasing the parameter ' $\mathrm{k}_{2}$ '. These results show that enhancing the parameter " $\mathrm{k}_{2}$ " aids the formation of complex $\mathrm{C}_{1}$ which, in turn, enhances the rate of 'DAG' metabolism. These results also reflect that complex $\mathrm{C}_{1}$ directly participates in the phosphorylation of 'DAG' to 'PA' and, therefore, its concentration is critical for regulating the "DAG" homeostasis.

\section{Discussion}

Dysregulation in cardiac contractile function is a fundamental characteristic of heart failure and has been linked to impaired circulation and fluid homeostasis [24,25]. Chemical agents called positive inotropes, which can enhance cardiac pump function during end-stage heart failure, may provide an attractive therapeutic strategy [24,26-28]. Previous work in this field has led to the discovery of agents which can clearly improve contractility in both acute and chronic heart failure [24,26-28]. Unfortunately, these agents are associated with high mortality rates, possibly due to desensitization of the entire $\beta$-adrenergic system [25-27]. Thus, in many clinical settings, the safety of traditional inotropes is controversial [27-30]. Therefore, there is a critical need for heart failure research to focus on finding therapeutics that are milder alternatives to positive inotropes and do not come with a high risk of $\beta$-adrenergic system desensitization $[9,10,29,30]$. Animal models for heart failure indicate that pharmacological inhibition of PKC $\alpha$ may have therapeutic potential, as agents that regulate PKC $\alpha$ appear to be safer, with milder side effects compared to cAMP elevating agents $[8,12,28-35]$. The enhanced safety profile of PKC $\alpha$ inhibitors is probably due to their target mechanisms of action. These agents act at the level of sarcoplasmic reticulum and myofilament proteins [9-12,35]. Unlike traditional inotropic therapies, inotropes that work through inhibition of PKC $\alpha$ are not thought to engage upstream signaling. Thus, treatment with these agents may not result in $\beta$-adrenergic system desensitization. Additionally, it remains entirely possible that PKC $\alpha$ inhibition may also affect the 
heart in ways other than alterations in contractile performance. PKC $\alpha$ inhibition may positively affect ventricular remodeling and the activity of other stress signaling pathways [11,35].

This study proposes PKC $\alpha$ may partially modulate the transition of initial compensatory responses to impaired cardiac function. PKC $\alpha$-mediated changes and impairments to the calcium cycle and/or causing stiffness patterns in myofilament proteins may regulate the key molecular mechanisms responsible for the transition to contractile dysfunction and development of arrhythmias. Experimental evidence provides a strong causal link between PKC $\alpha$ and impaired ventricular systolic and diastolic functions $[8,11,12,35]$. Clinical observations show that PKC $\alpha$ inhibition significantly improved cardiac output in heart failure models [8-12,35]. In this study, I investigated the general hypothesis that, in G $\alpha$ q-induced models of heart failure, PKC $\alpha$ may regulate its own activity through a positive feedback loop. The regulatory model proposed in this work is based on the observation that, during normal cellular conditions (low-intensity stimulation), DGK $\zeta$ molecules can regulate local PKC $\alpha$ activity by metabolizing its activator DAG in a spatially-selective manner $[19,20]$. However, during pathological conditions (high-intensity stimulation), local DAG generation may outpace the inhibitory activity of DGK $\zeta$, thus activating PKC $\alpha$. Once active PKC $\alpha$ molecule deactivates DGK $\zeta$ through phosphorylation, the molecule induces its own activation $[19,20]$. Based on these observations, I propose a two-compartment regulatory model of local DAG signaling in cardiomyocytes.

The two-compartment model proposed in this study accounts for a sequence of complex events. The model accounts for DAG-induced translocation of PKC $\alpha$ and DGK $\zeta$, blocking of PKC $\alpha$ translocation through DGK $\zeta$, formation of biochemical complex $C_{1}$ between PKC $\alpha$ and DGK $\zeta$, DAG removal through $C_{1}$-catalyzed metabolism, DAG-induced activation of $C_{1}$, and activation of PKC $\alpha$. In addition, the model accounts for deactivation of DGK $\zeta$, re-translocation of active PKC $\alpha$ from membrane to cytosol, eventual deactivation of PKC $\alpha$ in the cytosol to its dormant form, and dephosphorylation and re-translocation of DGK $\zeta$ back to the cytosol. Through incorporating these molecular details, the proposed model provides a mechanistic understanding of how PKC $\alpha$ and DGK $\zeta$ molecules may interact within cardiomyocytes during healthy states and during heart failure disease states.

The proposed local regulatory model of DAG signaling in G $\alpha q$-induced heart failure is composed of two functional loops. The first is a feedback loop exerting negative influence. The second is a molecular loop exerting positive influence on local DAG signaling. The negative feedback loop is generated due to two signaling events. The first signaling event is the DAG-modulated translocation of PKC $\alpha$ and DGK $\zeta$ from the cytosol to the plasma membrane. The second signaling event is the formation of a biochemical complex " $\mathrm{C}_{1}$ " at the plasma membrane. The positive effects on local DAG concentration are generated due to three molecular events. The first is the DAG-modulated activation of complex " $\mathrm{C}_{1}$ " and its subsequent dissociation into $\mathrm{PKC}_{\mathrm{I}} \alpha$ Active and $\mathrm{DGK}_{\mathrm{I}} \zeta$. The second molecular event is the phosphorylation and deactivation of $\mathrm{DGK}_{\mathrm{I}} \zeta$ by $\mathrm{PKC}_{\mathrm{I}} \alpha^{\text {Active }}$. The third molecular event is the degradation of $\mathrm{PKC}_{\mathrm{II}} \alpha^{\text {Active }}$ from the cytosol.

DAG is a membrane lipid that has a well-documented role in modulating the activity of numerous proteins, including PKC, nPKC, RasGRPs and receptor channel proteins. Due to its broad range of effects, DAG signaling is necessary for normal cellular function. However, DAG signaling must be exquisitely regulated [36,37]. Observations indicate that persistent DAG accumulation may induce malignant cellular transformations and therefore any dysregulation in the molecular apparatus which regulates its metabolism may result in a disease state [36]. A multitude of detrimental effects such as alterations in insulin signaling, apoptosis, endoplasmic reticulum stress and mitochondrial dysfunction have been linked to persistent intracellular lipid accumulation [38-45]. As the heart requires a lot of energy for proper function, lipids serve as a source of this energy [37]. However, persistent accumulation of lipids in the heart can lead to physiological dysfunction and death [37]. DAG accumulation has been linked to cardiac dysfunction $[39,44]$. The exact cause of lipid toxicity, however, is not clear. It is believed that accumulation of lipids disrupts $\beta$-AR signaling through PKC activation, thus altering normal physiological responses to stress [37]. This study assumes that, during normal physiological function, DAG homeostasis at the plasma membrane is regulated through a negative feedback signal (Figure 1; 
Green color pulse and signaling loop). The balance between local DAG biosynthesis and its removal is probably disrupted during this disease state. The disruption in DAG homeostasis is due to a positive feedback signal (Figure 1; Red color pulse and signaling loop). During normal cellular functions, the amount of DAG generated at the local plasma membrane site is quickly removed through $\mathrm{C}_{1}$-mediated metabolism of local DAG. In the pathological state, however, local rates of DAG biosynthesis may far exceed the $\mathrm{C}_{1}$-mediated removal rate, leading to a net accumulation of DAG at specific locations in the plasma membrane compartment. These accumulations then activate and destabilize the complex $C_{1}$. Once active, $C_{1}{ }^{A}$ dissociates into $\mathrm{PKC}_{\mathrm{I}} \alpha^{\text {Active }}$ and $D \mathrm{DK}_{\mathrm{I}} \zeta$. The $\mathrm{PKC}_{\mathrm{I}} \alpha^{\text {Active }}$ molecule further enhances the positive feedback effect by phosphorylating and deactivating $\mathrm{DGK}_{\mathrm{I}} \zeta$. According to the proposed model, when local DAG levels and DAG biosynthesis rates are low, a negative feedback loop is induced to facilitate DAG removal from local sites. In contrast, a higher local DAG biosynthesis rate leads to a positive feedback signal. The structure of the proposed model suggests that, during the disease state, DAG may lead to its own persistent accumulation through a positive feedback signaling loop.

This study also explains that, during normal physiological function, the precise spatiotemporal regulation of "DAG" homeostasis may not principally depend on increased activity of its effector molecules PKC $\alpha$ and DGK $\zeta$. Instead, the proposed model suggests that DAG homeostasis is maintained through agonist-dependent, site-specific migration of effector molecules from the cytosol to the plasma membrane. Accordingly, DAG signaling capacity at the plasma membrane is critically dependent on the migration characteristics of effector molecules (DAG-dependent) to the plasma membrane and the localization, anchoring, and activation state of these effectors at the plasma membrane. The model proposes a delicate balance between the membrane translocation of effector molecules and their return to the cytosol that directly influences the amplitude and duration of DAG signaling at the plasma membrane. The results presented here indicate the possibility of a DAG-modulated functional coupling between PKC $\alpha$ and DGK $\zeta$. These simulated results are based on experimental observations made in cardiomyocytes. The Ang-II-induced membrane translocation of PKC $\alpha$ has been observed [11-15]. At least one previous experimental study [12] indicates that inhibiting PKC $\alpha$ translocation in $\mathrm{G}_{\alpha q}$-induced systolic and diastolic dysfunction may provide a mild therapeutic advantage during heart failure. Furthermore, experimental observations indicate enhanced DGK $\zeta$ expression in infracted hearts [46]. These results reflect that agonist-mediated translocation of PKC $\alpha$ and DGK $\zeta$ is modulated by DAG concentration at the plasma membrane. This simulation mimics these translocation events by modeling them through simple kinetic steps (Materials and Methods, Equations (2) and (4)). The rate constants of these kinetic steps are described by a simple proportional function of DAG concentration (Supplementary Material 1; Figure S4 and Supplementary Material 2; Table S1). This ad-hoc functional arrangement was developed for the sake of simplicity. The assumptions behind this mathematical expression are a simplified model of translocation events, and it may be argued that the simplifying assumptions render the model unrealistic. However, computational modeling requires accounting for complex molecular details involved using simplifying assumptions. Some of these complex molecular details are $\mathrm{Ca}^{2+} / \mathrm{DAG}$ release and diffusion, DAG and $\mathrm{Ca}^{2+}$ binding to certain domains, the possible interaction of PKC $\alpha$ and DGK $\zeta$ with translocating rails and scaffolding proteins [46], formation of macromolecular complexes [19,20], and precise anchoring at specific sites/domains. Modeling all these complex molecular details is beyond the scope of this study. The model proposed here is a representation of PKC $\alpha$ and DGK $\zeta$ translocation that may neglect many important molecular details, but it was chosen to elucidate the influence of DAG concentration on PKC $\alpha$ and DGK $\zeta$ migration from the cytosol to the plasma membrane through a simple mathematical expression.

As mentioned above, the membrane translocation events of PKC $\alpha$ and DGK $\zeta$ in this study are described through first order kinetic functions (Table S1). The rate constants of these functions were obtained by fitting the translocation kinetics $[13,20,45,46]$. Interestingly, experimental evidence supports a membrane-directed PKC $\alpha$ translocation in G $\alpha$-induced heart failure ${ }^{13}$. Evidence, also shows prolonged elevation of DGK $\zeta$ in post MI hearts [46]. Additionally, data from T cells is also 
used within this study to further validate the description of PKC $\alpha$ and DGK $\zeta$ translocations from the cytosol to the cell membrane [20,45]. Interestingly, MI is followed by an inflammatory reaction, and observations from previous study suggest that elevated DGK expression in the infracted area is due to infiltrating macrophages and granulocytes [46]. One limitation of the first order description of PKC $\alpha$ translocation presented here is its dependence on DAG rather than on calcium $\mathrm{Ca}^{+2}$. PKC $\alpha$ translocation is mainly driven by calcium binding; the $\mathrm{C}_{2}$ domain is the main stimulating factor for enzyme migration. However, this assumption was made because $\mathrm{DAG}$ and $\mathrm{IP}_{3}$ generation precedes $\mathrm{Ca}^{+2}$ release. In a PKC-independent manner, DAG generation stimulates extracellular $\mathrm{Ca}^{+2}$ entry via the transient receptor potential canonical $3\left(\mathrm{TRPC}_{3}\right)$ channel in sarcolemma [47]. This $\mathrm{Ca}^{+2}$ release is independent of intracellular calcium stores [47]. Thus, the assumption relating to DAG-dependent translocation may have some physiological basis. The assumption exists within the proposed model mainly for the purpose of simplification.

The biological function of the PKC $\alpha$ molecule in the proposed model is regulated through a four-step cycle [11,22]. The four steps within this model are translocation, activation, redistribution/ re-translocation and deactivation. The model assumes that inactive but catalytically competent $\mathrm{PKC}_{\mathrm{II}} \alpha$ is stored in the cytosol. De novo synthesis generates a rather unstable naïve form of $\mathrm{PKC}_{\mathrm{II}} \alpha$ which constitutively undergoes a sequence of ordered priming and autophosphorylations. This sequence produces a mature, inactive, phosphatase-resistant, and proteasome-resistant molecule [22]. These phosphorylations are essential for PKC $\alpha$ stability and catalytic competence [22]. The PKC life cycle is complex and modulated through precise and tightly-coupled molecular events [22]. Evidence suggests that, before PKC become responsive to second messengers, it must first be phosphorylated at three conserved positions [22]: Thr500, Thr641 and Ser660. This model does not attempt to model de novo synthesis or the subsequent processes of enzyme maturation through phosphorylation. This study assumes the cytosol contains sufficient amounts of mature, second-messenger-responsive $\mathrm{PKC}_{\mathrm{II}} \alpha$ enzyme. In these simulations, this is modeled by setting the initial conditions such that only the concentration of $\mathrm{PKC}_{\mathrm{II}} \alpha$ is non-negligible. The concentration of all other forms of $\mathrm{PKC} \alpha$ is initially set at negligibly small values.

Here, the time scales of results presented from in silico experiments (Figures 3-7) are within 20-50 min, which seems to be in contrast to much longer time scales of experimental data (3-21 days) $[13,46]$. However, this model was validated against data from animal models [13,46] on the scale of days and weeks as shown in Supplementary Materials (Figures S5-S8). Thus, this model shows that though the setting of heart failure is complex, a simplified model could be used to provide some mechanistic basis. Here, we are not showing the heart failure time scales of years (in humans) however, our model is able to generate activation and translocation data even at that time scales. This work also shows that overexpression of DGK $\zeta$ can reduce DAG-induced PKC $\alpha$ activation (Figure 4) [13-15]. Angiotensin II-induced local DAG generation stimulates the translocation of PKC $\alpha$ from the cytosol to the cellular membrane. As explained above, this translocation event is modeled through a simple proportionality function of DAG concentration. However, experimental observations also show that DGK $\zeta$ overexpression restricts PKC $\alpha$ migration from the cytosol to the membrane compartment [13-15]. Here, the inverse phenomenon is modeled through $C_{1}$-mediated metabolism of DAG. Since the translocation rate of $P K C \alpha$, $\lambda_{3}$, is directly proportional to DAG concentration, any reduction in DAG concentration at the plasma membrane will reduce PKC $\alpha$ translocation to the plasma membrane. This simplistic description of complex interactions between DGK $\zeta$ and PKC $\alpha$ molecules was chosen for simplicity. As overexpression of $\mathrm{DGK} \zeta$ is controlled in silico, it produces a dose-response curve showing dose-dependent inhibition of PKC $\alpha$ translocation (Figure 4a). These results are consistent with previous experimental observations linking DGK $\zeta$ overexpression to reduction in PKC $\alpha$ translocation in cardiomyocytes [13]. These results also explain why survival after MI is improved if cardiac-specific overexpression of DGK $\zeta$ is

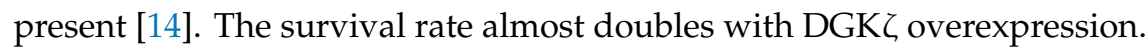

The results of this study indicate a role for $\mathrm{PKC}_{\mathrm{I}} \alpha^{\text {Active }}$ re-translocation to the cytosol (Figure 5). According to the model, once PKC $\alpha$ is active, it is not available to form complex $C_{1}$ and participate 
in DAG degradation. $\mathrm{PKC}_{\mathrm{I}} \alpha^{\text {Active }}$ re-translocation is necessary for the PKC $\alpha$ cycle to function and necessary for complex $C_{1}$ formation. The results of this study show that blocking the re-translocation process increases DAG levels (Figure 5). This model shows that dose-dependent blocking of re-translocation leads increased DAG in the membrane compartment (Figure 5b, Inset). These results explain the role of PKC $\alpha$ shuttling in maintaining DAG homeostasis.

While this model is based upon experimental results, its assumptions, as well as its consequences, require further testing. One of the key assumptions in the proposed translocation-dependent model is that local DAG generation is stimulated through agents such as angiotensin II or phenylephrine. This assumption is supported by observation that, in cardiomyocytes, Ang II and phenylephrine induced the local generation of DAG and subsequent translocation of PKC $\alpha$ from the cytosol to the plasma membrane $[7,13-15,48-50]$. Interestingly, other stimulating agents have only a small or negligible effect on PKC $\alpha$ translocation in cardiomyocytes [13]. This indicates that $\mathrm{CPKC}$ translocation in cardiomyocytes is highly dependent on the stimulating pathways and is consistent with the assumption that local DAG generation in response to angiotensin II-mediated activation of GPCR pathway is regulated through a complex process [48]. This model does not attempt to model this complex process. The model uses stimulating variable i.e., parameter $\mathrm{S}_{1}$ to mimic the GPCR agonist angiotensin II. A brief pulse of parameter $S_{1}$ at varying intensity represents the influence of angiotensin II on local DAG biosynthesis [48]. This simple approach ignores the complex details of angiotensin II-induced DAG biosynthesis [48]. Furthermore, the stimulation pulse was only applied for a 3-minute duration (Figures 3-7 and Figures S1-S3), that is why both PKC $\alpha$ and DGK $\zeta$ return to basal levels after $10 \mathrm{~min}$. This is in contrast to previous observations, showing significant PKC $\alpha$ elevation in angiotensin II-treated WT mice for 14 days [13]. This difference is due to the duration of pulse stimulation as shown in a Figure S5, where pulse is applied for 30 min leading to persistence of DAG and PKC $\alpha$. In conclusion, a two-compartment model was developed for regulating DAG homeostasis in Ang II-induced heart failure. This computational model is a promising tool to study mechanisms of DAG regulation in the context of heart failure. This model may be used to identify novel therapeutic targets with the aim of improving survival and quality of life outcomes in heart failure patients.

\section{Materials and Methods}

\subsection{Biochemical Reactions}

The biochemical interactions described in this study include G $\alpha$ q-induced local DAG-PKC $\alpha-$ DGK $\zeta$ signaling in cardiomyocytes [12-16]. The computationally-modeled interactions of this local molecular loop (Figures 1 and 2) are based on standard Michaelis-Menten kinetics. The following sets of biochemical reactions are used to describe the molecular interactions of other molecules with this loop. The dynamic variables used are: (1) DAG, representing second messenger diacylglycerol, (2) DGK $\zeta$ representing diacylglycerol kinase, (3) PKC $\alpha$, representing the $\alpha$ isoform of protein kinase C. A subscript I represents the concentration in the first compartment, which is the plasma membrane. Subscript II denotes the concentration in the second compartment, which is the cytosol. Superscript A represents the activated form of a molecule. Subscript $P$ represents the phosphorylated form of a molecule. The phosphatase $\mathrm{P}$ is approximated as a fixed parameter. The parameter $\mathrm{S}_{1}$ denotes AngII-induced stimulation, leading to the rapid generation of DAG.

$$
\begin{aligned}
& \mathrm{S}_{1} \stackrel{\mathrm{k}_{1}}{\rightarrow} \mathrm{DAG} \\
& \mathrm{PKC}_{\mathrm{II}} \alpha \stackrel{\lambda_{0}=\mathrm{f}(\mathrm{DAG})}{\rightarrow} \mathrm{PKC}_{\mathrm{I}} \alpha \\
& \mathrm{PKC}_{\mathrm{I}} \alpha \stackrel{\lambda_{00}}{\rightarrow} \mathrm{PKC}_{\mathrm{II}} \alpha \\
& \mathrm{DGK}_{\mathrm{II}} \zeta^{\lambda_{5}=\mathrm{f}(\mathrm{DAG})} \mathrm{DGK}_{\mathrm{I}} \zeta
\end{aligned}
$$




$$
\begin{aligned}
& \mathrm{DGK}_{\mathrm{I}} \zeta \stackrel{\lambda_{55}}{\rightarrow} \mathrm{DGK}_{\mathrm{II}} \zeta \\
& \mathrm{DGK}_{\mathrm{I}} \zeta+\mathrm{PKC}_{\mathrm{I}} \alpha \underset{\mathrm{k}_{3}}{\stackrel{\mathrm{k}_{2}}{\rightleftarrows}} \mathrm{C}_{1} \\
& \mathrm{C}_{1}+\mathrm{DAG} \underset{\mathrm{k}_{5}}{\stackrel{\mathrm{k}_{4}}{\rightleftarrows}} \mathrm{C}_{1}^{\mathrm{A}} \\
& \mathrm{C}_{1} \mathrm{~A} \stackrel{\mathrm{k}_{6}}{\rightarrow} \mathrm{PKC}_{\mathrm{I}} \alpha^{\mathrm{A}}+\mathrm{DGK}_{\mathrm{I}} \zeta \\
& \mathrm{PKC}_{\mathrm{I}} \alpha^{\mathrm{A}} \stackrel{\lambda_{3}}{\rightarrow} \mathrm{PKC}_{\mathrm{II}} \alpha^{\mathrm{A}} \\
& \mathrm{PKC}_{\mathrm{II}} \alpha^{\mathrm{A}} \stackrel{\lambda_{4}}{\rightarrow}[] \\
& \mathrm{PKC}_{\mathrm{II}} \alpha^{\mathrm{A}} \stackrel{\mathrm{k}_{0}}{\rightarrow} \mathrm{PKC}_{\mathrm{II}} \alpha \\
& \mathrm{DGK}_{\mathrm{I}} \zeta+\left[\mathrm{PKC}_{\mathrm{I}} \alpha\right]^{\mathrm{A}} \underset{\mathrm{k}_{8}}{\stackrel{\mathrm{k}_{7}}{\rightleftarrows}} \mathrm{C}_{2} \stackrel{\mathrm{k}_{9}}{\rightarrow}\left[\mathrm{PKC}_{\mathrm{I}} \alpha\right]^{\mathrm{A}}+\mathrm{DGK}_{\mathrm{I}} \zeta_{\mathrm{P}} \\
& \mathrm{C}_{1}+\mathrm{DAG} \underset{\mathrm{k}_{11}}{\stackrel{\mathrm{k}_{10}}{\rightleftarrows}} \mathrm{C}_{3} \stackrel{\mathrm{k}_{12}}{\rightarrow} \mathrm{C}_{1}+\mathrm{DAG}_{\mathrm{P}} \\
& \mathrm{DAG}_{\mathrm{P}}+\mathrm{P} \stackrel{\mathrm{k}_{13}}{\rightarrow} \mathrm{DAG}+\mathrm{P} \\
& \mathrm{DAGP}_{\mathrm{P}}+\mathrm{P} \stackrel{\mathrm{k} 14}{\rightarrow} \mathrm{P} . \mathrm{A} \\
& \mathrm{DGK}_{\mathrm{I}} \zeta_{\mathrm{P}}+\mathrm{P} \stackrel{\mathrm{k}_{15}}{\rightarrow} \mathrm{DGK}_{\mathrm{I}} \zeta+\mathrm{P}
\end{aligned}
$$

Signaling in this loop is initiated by local generation of DAG at the lipid membrane in angiotensin-stimulated cardiomyocytes. This generation of the DAG second messenger is described through Equation (1). DAG generation stimulates the migration of dormant and inactive $\mathrm{PKC}_{\mathrm{II}} \alpha$ from the cytosol to the lipid membrane. PKC $\alpha$ translocation is described in Equation (2). Here, the migration rate $\lambda_{0}$ is described through a function which is directly proportional to DAG concentration (Figure S4 and Table S1). $\mathrm{PKC}_{\mathrm{I}} \alpha$ is now an inactive $\alpha$-molecule in the lipid membrane compartment. $\mathrm{PKC}_{\mathrm{I}} \alpha$ then translocates back to the cytosol with a fixed migration rate $\lambda_{00}$, described in Equation (3). Here, $\mathrm{DGK}_{\mathrm{II}} \zeta$, which attenuates DAG signaling, also migrates from the cytosol to the plasma membrane in a DAG-dependent manner. This migration event is described in Equation (4). DGK $\mathrm{I}_{\mathrm{I}}$, the $\zeta$ molecule at the plasma membrane, can also translocate back to the cytosol, as described in Equation (5). Once at the plasma membrane, both $\mathrm{PKC}_{\mathrm{I}} \alpha$ and $\mathrm{DGK}_{\mathrm{I}} \zeta$ bind one another, forming a biochemical complex $C_{1}[19,20]$ This event is described in Equation (6). Under basal conditions, complex $C_{1}$ regulates local levels of DAG by metabolizing the second messenger through phosphorylation. These regulatory steps are modeled in Equations ((13)-(15)). However, upon stimulation, local DAG levels rise very sharply. These levels outpace the ability of complex $C_{1}$ to convert DAG to PA. The net accumulation of DAG activates complex $C_{1}$ through $\mathrm{PKC}_{\mathrm{I}} \alpha$ binding within $C_{1}[10,19,20]$. This activation event is described in Equation (7). The active form $C_{1}{ }^{A}$ is unstable and quickly dissociates into $P K C_{I} \alpha$ Active and $\mathrm{DGK}_{\mathrm{I}} \zeta$, as modeled in Equation $[19,20](8)$. In turn, $\mathrm{PKC}_{\mathrm{I}} \alpha^{\text {Active }}$ deactivates $\mathrm{DGK}_{\mathrm{I}} \zeta$ through phosphorylation [21]. This deactivation event is described in Equation (12). Through this event, PKC $\alpha$ prolongs its own activation. The active form of $\mathrm{PKC}_{\mathrm{I}} \alpha^{\text {Active }} \alpha$-isoform migrates from the plasma membrane to the cytosol [11] as described in Equation (9). Here, the migration rate $\lambda_{3}$ is set as a fixed parameter. Once inside the cytosol, the active form of $\mathrm{PKC}_{\mathrm{II}} \alpha^{\text {Active }}$ can either follow a degradation pathway as described in Equation (10) or undergo deactivation to its dormant form [11,22]. The fate of $\mathrm{PKC}_{\mathrm{II}} \alpha^{\text {Active }}$ is modeled in Equation (11). DGK $\zeta_{\mathrm{P}}$ dephosphorylation is described by Equation (16). 


\subsection{Induction}

During simulations, the angiotensin II-mediated activation of the GPCR pathway [7] is mimicked through a brief 3-minute pulse which leads to local biosynthesis of second messenger DAG in the membrane compartment, thus inducing the translocation and activation of DAG effector molecules.

\subsection{Temporal Dynamics}

The differential equations (Supplementary Material 3: Equations (17)-(28) resulting from the above interactions (Equations (1)-(16)) were integrated through nonlinear solvers using MATLAB (MathWorks). The dynamical coefficients' values were estimated from limited experimental data [11-23,46-50]. Unless otherwise stated, all of the molecular concentrations in the model are expressed as $\mathrm{pg} / \mathrm{ml}$ and time is represented in seconds.

Supplementary Materials: Supplementary materials can be found at http://www.mdpi.com/1422-0067/21/8/2561/s1. Funding: This study was funded by the author's own private funds.

Acknowledgments: Special thanks to M. Ashraf Ali Aziz, Ex. Chairman Department of Pathology, King Faisal Specialist Hospital and Research Center and Ex. Head Department of Anatomic Pathology Guards Hospital, Kingdom of Saudi Arabia for support and inspiration.

Conflicts of Interest: The authors declare no conflict of interest. The funders had no role in the design of the study; in the collection, analyses, or interpretation of data; in the writing of the manuscript, or in the decision to publish the results.

\section{References}

1. Ertl, G.; Gaudron, P.; Neubauer, S.; Bauer, B.; Horn, M.; Hu, K.; Tian, R. Cardiac dysfunction and development of heart failure. Eur. Heart J. 1993, 14 (Suppl. A), 33-37. [CrossRef] [PubMed]

2. Sun, Y. Myocardial repair/remodelling following infarction: Roles of local factors. Cardiovasc. Res. 2009, 81, 482-490. [CrossRef] [PubMed]

3. Schwinger, R.H.; Böhm, M.; Müller-Ehmsen, J.; Uhlmann, R.; Schmidt, U.; Stäblein, A.; Uberfuhr, P.; Kreuzer, E.; Reichart, B.; Eissner, H.J. Effect of inotropic stimulation on the negative force-frequency relationship in the failing human heart. Circulation 1993, 88, 2267-2276. [CrossRef] [PubMed]

4. Kurokawa, J.; Abriel, H. Neurohormonal regulation of cardiac ion channels in chronic heart failure. J. Cardiovasc. Pharm. 2009, 54, 98-105. [CrossRef] [PubMed]

5. Kehat, I.; Molkentin, J. Molecular Pathways Underlying Cardiac Remodeling During Pathophysiological Stimulation. Circulation 2010, 122, 2727-2735. [CrossRef] [PubMed]

6. Luo, M.; Anderson, M.E. Mechanisms of Altered $\mathrm{Ca}^{2+}$ Handling in Heart Failure. Circ. Res. 2013, 113, 690-708. [CrossRef]

7. Jarkko, P. Regulation of Cardiac Responses to Increased Load. Role of Endothelin-1, Angiotensin II and Collagen $X V$; Department of Pharmacology and Toxicology and Biocenter Oulu, University of Oulu, P.O.Box 5000, FIN-90014 University of Oulu, Finland: Oulu, Finland, 2002.

8. Liu, Q.; Chen, X.; Macdonnell, S.M.; Kranias, E.G.; Lorenz, J.N.; Leitges, M.; Houser, S.R.; Molkentin, J.D. Protein kinase C\{alpha\}, but not PKC \{beta\} or PKC $\{$ gamma\}, regulates contractility and heart failure susceptibility: Implications for ruboxistaurin as a novel therapeutic approach. Circ. Res. 2009, 105, 194-200. [CrossRef]

9. Michael, H.; Harvey, H.; Sven, T.P.; Matthew, C.K.; Raisa, K.; Andrew, N.C.; Thomas, F.K.; Timothy, E.H.; Gerald, W.D., II; Walter, J.K.; et al. Pharmacological- and Gene Therapy-Based Inhibition of Protein Kinase $C \alpha / \beta$ Enhances Cardiac Contractility and Attenuates Heart Failure. Circulation 2006, 114, 574-582.

10. Hambleton, M.; York, A.; Sargent, M.A.; Kaiser, R.A.; Lorenz, J.N.; Robbins, J.; Molkentin, J.D. Inducible and myocyte-specific inhibition of PKCalpha enhances cardiac contractility and protects against infarction-induced heart failure. Am. J. Physiol. Heart Circ. Physiol. 2007, 293, H3768-H3771. [CrossRef]

11. Steinberg, S.F. Cardiac Actions of Protein Kinase C Isoforms. Physiology 2012, 27, 130-139. [CrossRef] 
12. Hahn, H.S.; Marreez, Y.; Odley, A.; Sterbling, A.; Yussman, M.G.; Hilty, K.C.; Bodi, I.; Liggett, S.B.; Schwartz, A.; Dorn, G.W. Protein kinase C alpha negatively regulates systolic and diastolic function in pathological hypertrophy. Circ. Res. 2003, 93, 1111-1119. [CrossRef] [PubMed]

13. Arimoto, T.; Takeishi, Y.; Takahashi, H.; Shishido, T.; Niizeki, T.; Koyama, Y.; Shiga, R.; Nozaki, N.; Nakajima, O.; Nishimaru, K.; et al. Cardiac-specific overexpression of diacylglycerol kinase zeta prevents Gq protein-coupled receptor agonist-induced cardiac hypertrophy in transgenic mice. Circulation 2006, 113, 60-66. [CrossRef] [PubMed]

14. Niizeki, T.; Takeishi, Y.; Arimoto, T.; Takahashi, H.; Shishido, T.; Koyama, Y.; Goto, K.; Walsh, R.A.; Kubota, I. Cardiac-specific overexpression of diacylglycerol kinase zeta attenuates left ventricular remodeling and improves survival after myocardial infarction. Am. J. Physiol. Heart Circ. Physiol. 2007, 292, H1105-H1112. [CrossRef] [PubMed]

15. Harada, M.; Takeishi, Y.; Arimoto, T.; Niizeki, T.; Kitahara, T.; Goto, K.; Walsh, R.A.; Kubota, I. Diacylglycerol kinase zeta attenuates pressure overload-induced cardiac hypertrophy. Circ. J. 2007, 71, 276-282. [CrossRef]

16. Niizeki, T.; Takeishi, Y.; Kitahara, T.; Arimoto, T.; Ishino, M.; Bilim, O.; Suzuki, S.; Sasaki, T.; Nakajima, O.; Walsh, R.A.; et al. Diacylglycerol kinase-epsilon restores cardiac dysfunction under chronic pressure overload: A new specific regulator of Galpha(q) signaling cascade. Am. J. Physiol. Heart Circ. Physiol. 2008, 295, H245-H255. [CrossRef] [PubMed]

17. Bai, L.; Debra, S.R.; Stephen, M.P.; Matthew, K.T. Diacylglycerol kinases. Cell. Signal. 2004, 16, 983-989.

18. Takeishi, Y.; Goto, K.; Kubota, I. Role of diacylglycerol kinase in cellular regulatory processes: A new regulator for cardiomyocyte hypertrophy. Pharm. Ther. 2007, 115, 352-359. [CrossRef]

19. Luo, B.; Prescott, S.M.; Topham, M.K. Association of diacylglycerol kinase zeta with protein kinase C alpha: Spatial regulation of diacylglycerol signaling. J. Cell Biol. 2003, 160, 929-937. [CrossRef]

20. Gharbi, S.I.; Rincón, E.; Avila-Flores, A.; Torres-Ayuso, P.; Almena, M.; Cobos, M.A.; Albar, J.P.; Mérida, I. Diacylglycerol kinase $\zeta$ controls diacylglycerol metabolism at the immunological synapse. Mol. Biol. Cell 2011, 22, 4406-4414. [CrossRef]

21. Luo, B.; Prescott, S.M.; Topham, M.K. Protein Kinase C\{alpha\} Phosphorylates and Negatively Regulates Diacylglycerol Kinase \{zeta\}. J. Biol. Chem. 2003, 278, 39542-39547. [CrossRef]

22. Newton, A.C. Protein kinase C: Poised to signal. Am. J. Physiol. Endocrinol. Metab. 2010, 298 , E395-E402. [CrossRef] [PubMed]

23. Gao, T.; Brognard, J.; Newton, A.C. The phosphatase PHLPP controls the cellular levels of protein kinase C. J. Biol. Chem. 2008, 283, 6300-6311. [CrossRef] [PubMed]

24. Dorn, G.W.I.I.; Molkentin, J.D. Manipulating cardiac contractility in heart failure: Data from mice and men. Circulation 2004, 109, 150-158. [CrossRef] [PubMed]

25. Francis, G.S.; Tang, W.H. Pathophysiology of congestive heart failure. Rev. Cardiovasc. Med. 2003, 4, S14-S20.

26. Houser, S.R.; Margulies, K.B. Is depressed myocyte contractility centrally involved in heart failure? Circ. Res. 2003, 92, 350-358. [CrossRef]

27. Colucci, W.S.; Leatherman, G.F.; Ludmer, P.L.; Gauthier, D.F. Adrenergic inotropic responsiveness of patients with heart failure: Studies with intracoronary dobutamine infusion. Circ. Res. 1987, 61 (Suppl. I), I82-I86.

28. Feldman, A.M.; Bristow, M.R.; Parmley, W.W.; Carson, P.E.; Pepine, C.J.; Gilbert, E.M.; Strobeck, J.E.; Hendrix, G.H.; Powers, E.R.; Bain, R.P.; et al. Effects of vesnarinone on morbidity and mortality in patients with heart failure. Vesnarinone Study Group. N. Engl. J. Med. 1993, 329, 149-155. [CrossRef]

29. Chatterjee, K.; De Marco, T. Role of nonglycosidic inotropic agents: Indications, ethics, and limitations. Med. Clin. N. Am. 2003, 87, 391-418. [CrossRef]

30. Warner-Stevenson, L. Clinical use of inotropic therapy for heart failure: Looking backward or forward? Circulation 2003, 108, 492-497. [CrossRef]

31. Felker, G.M.; O'Connor, C.M. Inotropic therapy for heart failure: An evidence-based approach. Am. Heart J. 2001, 142, 393-401. [CrossRef]

32. Packer, M.; Gheorghiade, M.Y.; James, B.; Costantini, P.J.; Adams, K.F.; Cody, R.J.; Smith, L.K.; Van Voorhees, L.; Gourley, L.A.; Jolly, M.K.; et al. Withdrawal of digoxin from patients with chronic heart failure treated with angiotensin-converting- enzyme inhibitors. Radiance Study. N. Engl. J. Med. 1993, 329, 1-7. [CrossRef]

33. Digitalis Investigation Group. The effect of digoxin on mortality and morbidity in patients with heart failure. The Digitalis Investigation Group. N. Engl. J. Med. 1993, 336, 525-533. 
34. Braz, J.C.; Gregory, K.; Pathak, A.; Zaho, W.; Sahin, B.; Klevitsky, R.; Kimball, T.F.; Lorenz, J.N.; Nairn, A.C.; Liggett, S.B.; et al. PKC- $\alpha$ regulates cardiac contractility and propensity toward heart failure. Nat. Med. 2004, 10, 248-254. [CrossRef]

35. Pi, Y.; Walker, J.W. Diacylglycerol and fatty acids synergistically increase cardiomyocyte contraction via activation of PKC. Am. J. Phsiol. Heart Cir. Physiol. 2000, 279, H26-H34. [CrossRef] [PubMed]

36. Drosatos, K.; Bharadwaj, K.G.; Lymperopoulos, A.; Ikeda, S.; Khan, R.; Hu, Y.; Agarwal, R.; Yu, S.; Jiang, H.; Steinberg, S.F.; et al. Cardiomyocyte lipids impair $\beta$-adrenergic receptor function via PKC activation. Am. J. Physiol. Endocrinol. Metab. 2011, 300, E489-E499. [CrossRef] [PubMed]

37. Chavez, J.A.; Summers, S.A. Characterizing the effects of saturated fatty acids on insulin signaling and ceramide and diacylglycerol accumulation in 3T3-L1 adipocytes and C2C12 myotubes. Arch. Biochem. Biophys. 2003, 419, 101-109. [CrossRef] [PubMed]

38. Chiu, H.C.; Kovacs, A.; Ford, D.A.; Hsu, F.F.; Garcia, R.; Herrero, P.; Saffitz, J.E.; Schaffer, J.E. A novel mouse model of lipotoxic cardiomyopathy. J. Clin. Investig. 2001, 107, 813-822. [CrossRef] [PubMed]

39. Borradaile, N.M.; Buhman, K.K.; Listenberger, L.L.; Magee, C.J.; Morimoto, E.T.; Ory, D.S.; Schaffer, J.E. A critical role for eukaryotic elongation factor 1A-1 in lipotoxic cell death. Mol. Biol. Cell 2006, 17, 770-778. [CrossRef] [PubMed]

40. Turner, M.D. Fatty acyl CoA-mediated inhibition of endoplasmic reticulum assembly. Biochim. Biophys. Acta 2004, 1693, 1-4. [CrossRef]

41. Schrauwen, P.; Schrauwen-Hinderling, V.; Hoeks, J.; Hesselink, M.K. Mitochondrial dysfunction and lipotoxicity. Biochim. Biophys. Acta 2010, 1801, 266-271. [CrossRef]

42. Yu, C.; Chen, Y.; Cline, G.W.; Zhang, D.; Zong, H.; Wang, Y.; Bergeron, R.; Kim, J.K.; Cushman, S.W.; Cooney, G.J.; et al. Mechanism by which fatty acids inhibit insulin activation of insulin receptor substrate-1 (IRS-1)-associated phosphatidylinositol 3-kinase activity in muscle. J. Biol. Chem. 2002, 277, 50230-50236. [CrossRef] [PubMed]

43. Gopee, N.V.; Sharma, R.P. Selective and transient activation of protein kinase C alpha by fumonisin B1, a ceramide synthase inhibitor mycotoxin, in cultured porcine renal cells. Life Sci. 2004, 74, 1541-1559. [CrossRef] [PubMed]

44. Abramovici, H.; Hogan, A.B.; Obagi, C.; Topham, M.K.; Gee, S.H. Diacylglycerol kinase-zeta localization in skeletal muscle is regulated by phosphorylation and interaction with syntrophins. Mol. Biol. Cell 2003, 14, 4499-4511. [CrossRef] [PubMed]

45. Gharbi, S.I.; Avila-Flores, A.; Soutar, D.; Orive, A.; Koretzky, G.A.; Albar, J.P.; Merida, I. Transient PKC $\alpha$ shuttling to the immunological synapse is governed by Diacylglycerol kinase $\zeta$ and regulates L-selection shedding. J. Cell Sci. 2013, 126, 2176-2186. [CrossRef]

46. Takeda, M.; Kagaya, Y.; Takahashi, J.; Sugie, T.; Ohta, J.; Watanabe, J.; Shirato, K.; Kondo, H.; Goto, K. Gene Expression and In Situ Localization of Diacylglycerol Kinase Isozymes in Normal and Infarcted Rat Hearts Effects of Captopril Treatment. Circ. Res. 2001, 89, 265-272. [CrossRef]

47. Sabourin, J.; Antigny, F.; Robin, E.; Frieden, M.; Raddatz, E. Activation of Transient Receptor Potential Canonical 3 (TRPC3)-mediated Ca+2 Entry by A1 Adenosine Receptor in Cardiomyocytes Disturbs Atrioventricular Conduction. J. Biol. Chem. 2012, 287, 26688-26701. [CrossRef]

48. Sadoshima, J.; Izumo, S. Signal Transduction Pathways of Angiotensin-II Induced c-fos Gene Expression in Cardiac Myocytes In-Vitro Roles of Phospholipid-Derived Second Messengers. Circ. Res. 1993, 73, 424-438. [CrossRef]

49. Topham, M.K.; Bunting, M.; Zimmerman, G.A.; McIntyre, T.M.; Blackshear, P.J.; Prescott, S.M. Protein kinase $\mathrm{C}$ regulates the nuclear localization of diacylglycerol kinase-Zeta. Nature 1998, 394, 697-700. [CrossRef]

50. Goto, K.; Kondo, H. A 104-kDa diacylglycerol kinase containing ankyrin-like repeats localizes in the cell nucleus. Proc. Natl. Acad. Sci. USA 1996, 93, 11196-11201. [CrossRef]

(C) 2020 by the author. Licensee MDPI, Basel, Switzerland. This article is an open access article distributed under the terms and conditions of the Creative Commons Attribution (CC BY) license (http://creativecommons.org/licenses/by/4.0/). 